\title{
Shortest Paths on Polyhedral Surfaces and Terrains*
}

\author{
Siu-Wing Cheng \\ HKUST \\ Hong Kong \\ scheng@cse.ust.hk
}

\author{
Jiongxin Jin \\ Google Inc. \\ 651 N 34th St, Seattle, WA, 98103 \\ jamesjjx@google.com
}

\begin{abstract}
We present an algorithm for computing shortest paths on polyhedral surfaces under convex distance functions. Let $n$ be the total number of vertices, edges and faces of the surface. Our algorithm can be used to compute $L_{1}$ and $L_{\infty}$ shortest paths on a polyhedral surface in $O\left(n^{2} \log ^{4} n\right)$ time. Given an $\varepsilon \in(0,1)$, our algorithm can find $(1+\varepsilon)$ approximate shortest paths on a terrain with gradient constraints and under cost functions that are linear combinations of path length and total ascent. The running time is $O\left(\frac{1}{\sqrt{\varepsilon}} n^{2} \log n+n^{2} \log ^{4} n\right)$. This is the first efficient PTAS for such a general setting of terrain navigation.
\end{abstract}

\section{Categories and Subject Descriptors}

F.2.2 [Analysis of Algorithms and Problem Complexity]: Nonnumerical Algorithms and Problems - Geometrical problems and computations

\section{General Terms}

Algorithms, Theory

\section{Keywords}

shortest path, convex distance function, polyhedral surface, terrain

\section{INTRODUCTION}

Finding shortest paths is a classical geometric optimization problem. In recent years, the spatial database and geographical information system communities show interest in the shortest path problem on terrains under anisotropic cost models, i.e., the path cost at any point on the terrain depends on the travel direction $[10,12,15,17]$. The motivations are two-fold. First, when planning a roadway or

\footnotetext{
* Research supported by the Research Grant Council, Hong Kong, China (project no. 611812).

Permission to make digital or hard copies of all or part of this work for personal or classroom use is granted without fee provided that copies are not made or distributed for profit or commercial advantage and that copies bear this notice and the full citation on the first page. Copyrights for components of this work owned by others than the author(s) must be honored. Abstracting with credit is permitted. To copy otherwise, or republish, to post on servers or to redistribute to lists, requires prior specific permission and/or a fee. Request permissions from Permissions@acm.org. STOC'14, May 31-June 03 2014, New York, NY, USA Copyright is held by the owner/author(s). Publication rights licensed to ACM. ACM 978-1-4503-2710-7/14/05 ...\$15.00 http://dx.doi.org/10.1145/2591796.2591821.
}

hiking on a terrain, it is impossible to ascend or descend along slopes that are too steep. Second, the cost of a subpath may depend on the its slope. Anisotropic cost models on polyhedral surfaces also relate to or generalize previous results in the algorithm community: the shortest path problem on polyhedral surfaces $[6,13,16]$, the weighted region problem $[4,14]$, the anisotropic shortest path problem in the plane $[8,9]$, and the consideration of total ascent or descent of paths on a terrain [5]. The shortest descending path problem $[1,2,7]$ and the shortest gently descending path problem [3] are special cases obtained by enforcing particular gradient constraints.

For Euclidean shortest paths on a polyhedral surface of $n$ vertices, edges and faces, Mitchell et al. [13] presented an algorithm that runs in $O\left(n^{2} \log n\right)$ time, which was subsequently improved by Chen and Han [6] to $O\left(n^{2}\right)$. Varadarajan and Agarwal [16] proposed two approximation algorithms that run in subquadratic time: $7(1+\varepsilon)$ - and $15(1+\varepsilon)$ approximate shortest paths can be found in $O\left(n^{5 / 3} \log ^{5 / 3} n\right)$ and $O\left(n^{8 / 5} \log ^{8 / 3} n\right)$ time, respectively.

In the weighted region problem, each face $f$ has a weight $w_{f}$ and the subpath cost within $f$ is $w_{f}$ times the subpath length. A shortest path may bend when crossing edges (which also happens under anisotropic cost models). Mitchell and Papadimitriou [14] presented an algorithm for planar weighted regions that runs in $O\left(n^{8} \log (N W / \delta)\right)$ time, where $N$ is the largest integer coordinate, $W$ is the ratio of the maximum weight to the minimum weight, and $\delta$ is a precision parameter. Aleksandrov et al. [4] developed an algorithm for polyhedral surfaces that has a running time linear in $n$ and dependent on some geometric parameters.

Cheng et al. [8] proposed a $(1+\varepsilon)$-approximation algorithm for the anisotropic shortest path problem in a planar subdivision in which every face has a convex distance function. Later, a data structure was developed to answer $(1+\varepsilon)$-approximate anisotropic shortest path queries [9].

De Berg and van Kreveld [5] studied some path query problems on terrains with height constraints, and they posed the optimization of path length and total ascent as an open problem. There are $(1+\varepsilon)$-approximate algorithms for the shortest descending path problem [1] and the shortest gently descending path problem [3] that have running times dependent on some geometric parameters. Recently, we developed a $(1+\varepsilon)$-approximate shortest descending path algorithm that runs in $O\left(n^{4} \log (n / \varepsilon)\right)$ time [7].

This paper presents an algorithm for a shortest path problem on a polyhedral surface, which we call the PoLYPATH problem. Each face $f$ is associated with a convex polygon 
$H_{f}$ that induces a convex distance function $d_{f}$. The length of a subpath in $f$ is measured using $d_{f}$. Given two points $s$ and $t$ on the polyhedral surface and an integer $m$, the goal is to find a shortest one among all paths that have at most $m$ links and no critical refraction at any surface edge. ${ }^{1}$ The latter constraint can be removed if $d_{f}(p, q)=d_{g}(p, q)$ for every two adjacent faces $f$ and $g$ and every two points $p, q \in f \cap g$. Our algorithm runs in $O\left(h m n \log m n+m n \log ^{2} m \log ^{2} h m\right)$ time, where $h$ is the maximum size of the convex polygons associated with the faces. It follows that an $L_{1}$ or $L_{\infty}$ shortest path on a polyhedral surface can be computed in $O\left(n^{2} \log ^{4} n\right)$ time.

On terrains, for every constant $c_{1}>0$ and every constant $c_{2} \geq 0$, we can optimize $c_{1}$. Euclidean path length plus $c_{2}$. total ascent with a relative error $\varepsilon$ under gradient constraints. The total ascent is the total increase in heights of all ascending subpaths, which measures the energy spent in increasing the potential energy. The weighted sum of the path length and its total ascent gives rise to a convex distance function, which can be approximately induced by a convex polygon of size $O(1 / \sqrt{\varepsilon})$. This allows us to reduce the problem to an instance of POLYPATH such that $m=O(n)$ and $h=O(1 / \sqrt{\varepsilon})$. Gradient constraints are specified by the maximum ascent and descent gradients allowed in $\mathcal{T}^{2}$ This only changes the convex distance function slightly. Section 4 describes these reductions. In all, our algorithm can return a $(1+\varepsilon)$-approximate shortest path in $O\left(\frac{1}{\sqrt{\varepsilon}} n^{2} \log n+n^{2} \log ^{4} n\right)$ time, which makes it the first PTAS for such a general setting of terrain navigation. A $(1+\varepsilon)$-approximate shortest descending path can thus be computed in $O\left(\frac{1}{\sqrt{\varepsilon}} n^{2} \log n+n^{2} \log ^{4} n\right)$ time.

Our results address the problems in the applications $[10$, $12,15,17]$ mentioned earlier. A shortest path that satisfies gradient constraints is sought on a terrain in [12]. So our terrain algorithm is directly applicable. A main problem treated in $[10,15,17]$ is to optimize path length and penalize large slopes. As illustrated in Figure 5 in [10], one may model the cost function as a convex function in slope. Such a convex function translates to a convex distance function in a face. Therefore, if an upper bound $m$ on the number of links can be specified, our POLYPATH algorithm can be used to obtain a $(1+\varepsilon)$-approximation after approximating the convex distance function as in our terrain algorithm.

There are several difficulties in solving the POLYPATH problem. A locally shortest path $(L S P)$ for a sequence $\sigma$ of edges is a shortest path that crosses the edges in $\sigma$. An algorithm needs to extend an LSP from one face to the next. In the Euclidean and weighted region cases, the extension is determined locally by unfolding to a straight line and following Snell's law, respectively. In our case, we first discover how an LSP bends at a surface edge. In fact, an LSP may bend in various ways, and we focus on a special LSP in order to characterize the bending. However, the extension is not determined locally. In the Euclidean and weighted region cases, the local extension allows to construct a function to describe the costs of the LSPs that start from an interval on an edge, cross the edges in $\sigma$, and end at an interval on the

\footnotetext{
${ }^{1} \mathrm{~A}$ path makes a critical refraction at an edge $e$ if there are two non-collinear links such that one lies on $e$ and these two links meet at a node in the interior of $e$.

${ }^{2}$ The ascent and descent gradient bounds may be different, but they are the same for all faces.
}

last edge in $\sigma$. This is important as an algorithm cannot extend an infinite number of LSPs. We show that such a function can be constructed in our case by proving that LSPs are preserved under sliding, i.e. translating each segment of the path while keeping it parallel with the original one. Thus, after constructing one LSP for some edge sequence, the cost of another LSP with the same edge sequence is a function of the amount of sliding. Our third contribution is to compose a shortest path by combining shorter LSPs in a hierarchical fashion using Chen and Han's sequence tree [6], which yields the claimed running time.

\section{PRELIMINARIES}

Let $\mathcal{T}$ denote the input polyhedral surface with $n$ vertices, edges and faces. Without loss of generality, assume that each face of $\mathcal{T}$ is a triangle, and the source $s$ and the destination $t$ are vertices of $\mathcal{T}$. Each face $f$ of $\mathcal{T}$ is associated with a convex polygon $H_{f}$, which contains the origin, lies in a plane parallel to $f$, and induces the distance function $d_{f}$. We allow the origin to be on the boundary of $H_{f}$. The cost of a directed segment $p q \subset f$ is $\operatorname{cost}(p q)=$ $d_{f}(p, q)=\inf \left\{\lambda>0: \frac{1}{\lambda}(q-p) \in H_{f}\right\}$, which can be computed in $O\left(\log \left|H_{f}\right|\right)$ time by binary search.

We use $\vec{u}$ to denote a vector and $\hat{u}$ to denote the unit vector in the same direction as $\vec{u}$. Given $\vec{u}$ and $\vec{v}, \theta(\vec{u}, \vec{v})$ denotes the angle measured from $\vec{u}$ to $\vec{v}$ in counter-clockwise direction, which takes value in $[0,2 \pi)$. The inner product of $\vec{u}$ and $\vec{v}$ is denoted by $\langle\vec{u}, \vec{v}\rangle$.

All polygonal paths in this paper are oriented from their sources to their destinations. A link of a polygonal path is a maximal segment in a face or on an edge of $\mathcal{T}$, and its endpoints are called nodes. We assume that every node is either a vertex or a point in the interior of an edge because a node in the interior of a face can be removed by shortcutting without increasing the path cost. By the requirement of the PolyPATH problem, we can further assume that every node in the interior of an edge is a transversal node, that is, its two incident links lie in the interiors of two distinct faces.

Let $p_{i}, i \in[0, k]$, be the nodes in order along a path $P$. Let $\vec{v}_{i}=p_{i}-p_{i-1}$ for $i \in[1, k]$. The direction vector of $P$ is $\left(\hat{v}_{1}, \ldots, \hat{v}_{k}\right)$. We can specify $P$ as $\left(p_{0}, p_{1}, \ldots, p_{k}\right)$ or as $\left(p_{0},\left(\hat{v}_{1}, \ldots, \hat{v}_{k}\right)\right)$. The subpath of $P$ from a point $x$ to another point $y$ is denoted by $P[x, y]$. Define $\operatorname{cost}(P)=$ $\sum_{\text {face } f} \operatorname{cost}(P \cap f)$ and $\|P\|$ to be the length of $P$.

The edges that $P$ crosses in order is its edge sequence. It includes the edge containing $P$ 's destination but not the edge containing $P$ 's source. A path may have multiple edge sequences if its interior passes though a vertex. Suppose that the edges $e_{1}, e_{2}, \ldots, e_{k}$ are incident to a vertex $\nu$ in circular order. If a path moves from the face bounded by $e_{1}$ and $e_{k}$ to $\nu$ onward to the face bounded by $e_{i}$ and $e_{i+1}$, then one edge sequence contains the substring $e_{1} e_{2} \ldots e_{i}$, and another edge sequence contains the substring $e_{k} e_{k-1} \ldots e_{i+1}$. A shortest path from $s$ to $t$ is a shortest LSP over all edge sequences.

\section{SOLVING PolyPath}

We first characterize the LSPs by their direction vectors in Section 3.1. Then we propose an algorithm in Section 3.2 to solve the POLYPATH problem.

\subsection{Properties of LSPs}

Let $\sigma=\left(e_{1}, e_{2}, \ldots, e_{k}\right)$ be the edge sequence of some LSP 
that starts from a point $p_{0}$ on some face boundary and ends at a point $p_{k}$ on some other face boundary. Thus, $e_{i}$ and $e_{i+1}$ are distinct edges of the same face, and $e_{i}$ and $e_{i+2}$ do not bound the same face. Let $e_{0}$ denote an edge adjacent to $e_{1}$ that contains the source of the LSP. For $i \in[1, k]$, let $f_{i}$ denote the face bounded by $e_{i-1}$ and $e_{i}$.

For $i \in[1, k]$, define the positive and negative sides of a point on $e_{i}$ as follows. Orient $e_{i}$ to obtain a directed segment $a_{i} b_{i}$ so that $f_{i}$ and $f_{i+1}$ are on the left and right of $a_{i} b_{i}$, respectively. Let $\vec{e}_{i}$ denote the vector $b_{i}-a_{i}$. Given two points $p, q \in e_{i}$, we say that $q$ lies on the positive or negative side of $p$ if $\left\langle q-p, \vec{e}_{i}\right\rangle>0$ or $\left\langle q-p, \vec{e}_{i}\right\rangle<0$, respectively. The head and tail of the oriented $e_{i}$ are the positive and negative endpoints of $e_{i}$, respectively.

There may be multiple LSPs that start from $p_{0}$, end at $p_{k}$, and share an edge sequence $\sigma$. Let $P=\left(p_{0},\left(\hat{v}_{1}, \ldots, \hat{v}_{k}\right)\right)$ and let $Q=\left(p_{0},\left(\hat{w}_{1}, \ldots, \hat{w}_{k}\right)\right)$ be two such LSPs. We say that $\hat{v}_{i}$ is smaller than $\hat{w}_{i}$ if $\theta\left(\vec{e}_{i}, \hat{v}_{i}\right)<\theta\left(\vec{e}_{i}, \hat{w}_{i}\right)$. The canonical $L S P$ from $p_{0}$ to $p_{k}$ with edge sequence $\sigma$ is the LSP that has the lexicographically smallest direction vector. Intuitively, the canonical LSP hits every oriented $e_{i}$ at a point closest to its negative endpoint.

Lemma 3.1. Let $P=\left(p_{0}, p_{1}, \ldots, p_{k}\right)$ and $Q=\left(q_{0}=\right.$ $p_{0}, q_{1}, \ldots, q_{k}=p_{k}$ ) be two LSPs from $p_{0}$ to $p_{k}$ with the same edge sequence. If $P$ is a canonical $L S P$, then for $i \in[1, k-1]$, $q_{i}$ does not lie on the negative side of $p_{i}$.

Proof. Let $j$ be the smallest integer such that $p_{j} \neq q_{j}$. Since $P$ is the canonical LSP and $P\left[p_{0}, p_{j-1}\right]=Q\left[q_{0}, q_{j-1}\right]$, $p_{j}$ must be on the negative side of $q_{j}$. If for all $i>j$, $p_{i}=q_{i}$ or $p_{i}$ is on the negative side of $q_{i}$, then we are done. Otherwise, let $i$ be the smallest integer such that $p_{i}$ is on the positive side of $q_{i}$. Then $p_{i-1} p_{i}$ must cross $q_{i-1} q_{i}$, say at $x$. Since both $P$ and $Q$ are LSPs, $P\left[x, p_{k}\right]$ and $Q\left[x, p_{k}\right]$ are both LSPs and have the same cost, implying that

$$
\begin{aligned}
& \operatorname{cost}\left(P\left[p_{0}, p_{i-1}\right]\right)+d_{f_{i}}\left(p_{i-1} q_{i}\right)+\operatorname{cost}\left(Q\left[q_{i}, p_{k}\right]\right) \\
\leq & \operatorname{cost}\left(P\left[p_{0}, x\right]\right)+\operatorname{cost}\left(Q\left[x, p_{k}\right]\right)=\operatorname{cost}(P) .
\end{aligned}
$$

We obtain a new LSP $R=\left(p_{0}, p_{1}, \ldots, p_{i-1}, q_{i}, q_{i+1}, \ldots, q_{k}\right)$, where $\theta\left(\vec{e}_{i}, q_{i}-p_{i-1}\right)<\theta\left(\vec{e}_{i}, p_{i}-p_{i-1}\right)$. But then the direction vector of $R$ is lexicographically smaller than that of $P$, contradicting the assumption that $P$ is a canonical LSP.

We will characterize a canonical LSP via the derivative of its cost, which may not change smoothly as its destination moves. Thus, we define the derivative using limit and it depends on how the limit is approached. Recall that $\sigma=$ $\left(e_{1}, \ldots, e_{k}\right)$ and $e_{0}$ is an edge adjacent to $e_{1}$ containing the source $p_{0}$ of $P$. Let $\sigma_{i j}=\left(e_{i+1}, \ldots, e_{j}\right)$. For every point $p \in e_{i}$, define a function $C_{p, \sigma_{i j}}(x)$ to be the cost of an LSP with edge sequence $\sigma_{i j}$ from $p$ to a point $x \in e_{j}$. For every point $q \in e_{j}$, define the function $D_{q, \sigma_{i j}}(x)$ be the cost of an LSP with edge sequence $\sigma_{i j}$ from a point $x \in e_{i}$ to $q$. We use $x^{\prime} \rightarrow x^{+}$and $x^{\prime} \rightarrow x^{-}$to denote $x^{\prime}$ approaching $x$ from the positive and negative sides of $x$, respectively. Define:

$$
\begin{aligned}
& \partial C_{p, \sigma_{i j}}^{+}(x)=\lim _{x^{\prime} \rightarrow x^{+}} \frac{C_{p, \sigma_{i j}}\left(x^{\prime}\right)-C_{p, \sigma_{i j}}(x)}{\left\|x x^{\prime}\right\|}, \\
& \partial C_{p, \sigma_{i j}}^{-}(x)=\lim _{x^{\prime} \rightarrow x^{-}} \frac{C_{\sigma_{i j}(p, x)-C_{p, \sigma_{i j}}\left(x^{\prime}\right)}}{\left\|x x^{\prime}\right\|}, \\
& \partial D_{q, \sigma_{i j}}^{+}(x)=\lim _{x^{\prime} \rightarrow x^{+}} \frac{D_{q, \sigma_{i j}}\left(x^{\prime}\right)-D_{q, \sigma_{i j}}(x)}{\left\|x x^{\prime}\right\|}, \text { and } \\
& \partial D_{q, \sigma_{i j}}^{-}(x)=\lim _{x^{\prime} \rightarrow x^{-}} \frac{D_{q, \sigma_{i j}}(x)-D_{q, \sigma_{i j}}\left(x^{\prime}\right)}{\left\|x x^{\prime}\right\|} .
\end{aligned}
$$

LEMma 3.2. $C_{p, \sigma_{i j}}(x)$ and $D_{q, \sigma_{i j}}(x)$ are convex piecewise linear functions in $x$. If $y$ is on the positive side of $x$ in $e_{j}$, then $\partial C_{p, \sigma_{i j}}^{+}(y) \geq \partial C_{p, \sigma_{i j}}^{-}(y) \geq \partial C_{p, \sigma_{i j}}^{+}(x) \geq \partial C_{p, \sigma_{i j}}^{-}(x)$. If $y$ is on the positive side of $x$ in $e_{i}$, then $\partial D_{q, \sigma_{i j}}^{+}(y) \geq$ $\partial D_{q, \sigma_{i j}}^{-}(y) \geq \partial D_{q, \sigma_{i j}}^{+}(x) \geq \partial D_{q, \sigma_{i j}}^{-}(x)$.

Proof. $C_{p, \sigma_{i j}}(x)$ is the minimum of $\sum_{\ell=i+1}^{j} \operatorname{cost}\left(x_{\ell-1} x_{\ell}\right)$, where $x_{i}=p, x_{j}=x, x_{\ell} \in e_{\ell}$ for $\ell \in(i, j)$. The function $\operatorname{cost}\left(x_{\ell-1} x_{\ell}\right)$ is convex and piecewise linear in $x_{\ell-1}$ and $x_{\ell}$, so $C_{p, \sigma_{i j}}(x)$ is the minimization of the cross-section of a convex piecewise linear function. This implies the properties of $C_{p, \sigma_{i j}}, \partial C_{p, \sigma_{i j}}^{+}$and $\partial C_{p, \sigma_{i j}}^{-}$stated in the lemma. The same argument works for $D_{q, \sigma_{i j}}(x)$.

Our algorithm will form new LSP by concatenating shorter ones. It is clear that if we split a canonical LSP $\left(p_{0}, \ldots, p_{k}\right)$ at $p_{i}$, we obtain two shorter canonical LSPs. Lemma 3.3 below shows that the converse is true under some conditions.

Lemma 3.3. If a path $P=\left(p_{0}, p_{1}, \ldots, p_{k}\right)$ is a canonical $L S P$ with edge sequence $\sigma$, where $p_{i} \in e_{i}$, then the following conditions hold for every $i \in[1, k-1]$.

(i) $P\left[p_{0}, p_{i}\right]$ and $P\left[p_{i}, p_{k}\right]$ are canonical LSPs with edge sequences $\sigma_{0 i}$ and $\sigma_{i k}$, respectively.

(ii) $p_{i}$ is the positive endpoint of $e_{i}$ or

$$
\partial C_{p_{0}, \sigma_{0 i}}^{+}\left(p_{i}\right)+\partial D_{p_{k}, \sigma_{i k}}^{+}\left(p_{i}\right) \geq 0 .
$$

(iii) $p_{i}$ is the negative endpoint of $e_{i}$ or

$$
\partial C_{p_{0}, \sigma_{0 i}}^{-}\left(p_{i}\right)+\partial D_{p_{k}, \sigma_{i k}}^{-}\left(p_{i}\right)<0 .
$$

Conversely, if the conditions above hold for some $i \in[1, k-$ $1]$, then $P$ is a canonical LSP from $p_{0}$ to $p_{k}$ with edge sequence $\sigma$.

Proof. Suppose that $P$ is a canonical LSP. Then $P\left[p_{0}, p_{i}\right]$ and $P\left[p_{i}, p_{k}\right]$ are canonical LSPs as well. If $p_{i}$ is not the positive endpoint of $e_{i}$, pick a point $p_{i}^{\prime}$ on the positive side of $p_{i}$ and arbitrarily close to $p_{i}$. By the definition of the functions $\partial C_{p_{0}, \sigma_{0 i}}^{+}$and $\partial D_{p_{k}, \sigma_{i k}}^{+}$, we obtain

$$
\begin{aligned}
& C_{p_{0}, \sigma_{0 i}}\left(p_{i}^{\prime}\right)+D_{p_{k}, \sigma_{i k}}\left(p_{i}^{\prime}\right)-C_{p_{0}, \sigma_{0 i}}\left(p_{i}\right)-D_{p_{k}, \sigma_{i k}}\left(p_{i}\right) \\
= & \left(\partial C_{p_{0}, \sigma_{0 i}}^{+}\left(p_{i}\right)+\partial D_{p_{k}, \sigma_{i k}}^{+}\left(p_{i}\right)\right) \cdot\left\|p_{i}^{\prime} p_{i}\right\| .
\end{aligned}
$$

Since $P$ is an LSP, $C_{p_{0}, \sigma_{0 i}}\left(p_{i}^{\prime}\right)+D_{p_{k}, \sigma_{i k}}\left(p_{i}^{\prime}\right) \geq C_{p_{0}, \sigma_{0 i}}\left(p_{i}\right)+$ $D_{p_{k}, \sigma_{i k}}\left(p_{i}\right)$, which implies that $\partial C_{p_{0}, \sigma_{0 i}}^{+}\left(p_{i}\right)+\bar{\partial} D_{p_{k}, \sigma_{i k}}^{+}\left(p_{i}\right) \geq$ 0 . If $p_{i}$ is not the negative endpoint of $e_{i}$, we pick $p_{i}^{\prime} \in e_{i}$ on the negative side of $p_{i}$ and sufficiently close to $p_{i}$. Then,

$$
\begin{aligned}
& C_{p_{0}, \sigma_{0 i}}\left(p_{i}\right)+D_{p_{k}, \sigma_{i k}}\left(p_{i}\right)-C_{p_{0}, \sigma_{0 i}}\left(p_{i}^{\prime}\right)+D_{p_{k}, \sigma_{i k}}\left(p_{i}^{\prime}\right) \\
= & \left(\partial C_{p_{0}, \sigma_{0 i}}^{-}\left(p_{i}\right)+\partial D_{p_{k}, \sigma_{i k}}^{-}\left(p_{i}\right)\right) \cdot\left\|p_{i}^{\prime} p_{i}\right\| .
\end{aligned}
$$

By Lemma 3.1, $C_{p_{0}, \sigma_{0 i}}\left(p_{i}^{\prime}\right)+D_{p_{k}, \sigma_{i k}}\left(p_{i}^{\prime}\right)>C_{p_{0}, \sigma_{0 i}}\left(p_{i}\right)+$ $D_{p_{k}, \sigma_{i k}}\left(p_{i}\right)$, and therefore, $\partial C_{p_{0}, \sigma_{0 i}}^{-}\left(p_{i}\right)+\partial D_{p_{k}, \sigma_{i k}}^{-}\left(p_{i}\right)<0$.

Conversely, suppose that the three conditions are satisfied for some $i \in[1, k-1]$. Let $p_{i}^{\prime}$ be the intersection point between $e_{i}$ and the canonical LSP from $p_{0}$ to $p_{k}$ with edge sequence $\sigma$. If $p_{i}^{\prime}=p_{i}$, we are done. Suppose that $p_{i}^{\prime} \neq p_{i}$.

Consider the case of $p_{i}^{\prime}$ lying on the positive side of $p_{i}$. By Lemma 3.2, $C_{p_{0}, \sigma_{0 i}}$ and $D_{p_{k}, \sigma_{i k}}$ are convex functions. Therefore,

$$
\begin{aligned}
& C_{p_{0}, \sigma_{0 i}}\left(p_{i}^{\prime}\right) \geq C_{p_{0}, \sigma_{0 i}}\left(p_{i}\right)+\partial C_{p_{0}, \sigma_{0, i}}^{+}\left(p_{i}\right) \cdot\left\|p_{i} p_{i}^{\prime}\right\| \\
& D_{p_{k}, \sigma_{i k}}\left(p_{i}^{\prime}\right) \geq D_{p_{k}, \sigma_{i k}}\left(p_{i}\right)+\partial D_{p_{k}, \sigma_{i k}}^{+}\left(p_{i}\right) \cdot\left\|p_{i} p_{i}^{\prime}\right\|
\end{aligned}
$$


Combining these two inequalities and condition (ii) in the lemma gives

$$
C_{p_{0}, \sigma_{0 i}}\left(p_{i}^{\prime}\right)+D_{p_{0}, \sigma_{0 i}}\left(p_{i}^{\prime}\right) \geq C_{p_{0}, \sigma_{0 i}}\left(p_{i}\right)+D_{p_{0}, \sigma_{0 i}}\left(p_{i}\right),
$$

which shows that $P$ is also an LSP. However, $p_{i}$ is on the negative side of $p_{i}^{\prime}$, which is a contradiction to Lemma 3.1.

Consider the case of $p_{i}^{\prime}$ lying on the negative side of $p_{i}$. By the convexity argument again, we obtain

$$
\begin{aligned}
& C_{p_{0}, \sigma_{0 i}}\left(p_{i}^{\prime}\right) \geq C_{p_{0}, \sigma_{0 i}}\left(p_{i}\right)+\partial C_{p_{0}, \sigma_{0, i}}^{-}\left(p_{i}\right) \cdot\left\|p_{i} p_{i}^{\prime}\right\| \\
& D_{p_{k}, \sigma_{i k}}\left(p_{i}^{\prime}\right) \geq D_{p_{k}, \sigma_{i k}}\left(p_{i}\right)+\partial D_{p_{k}, \sigma_{i k}}^{-}\left(p_{i}\right) \cdot\left\|p_{i} p_{i}^{\prime}\right\|
\end{aligned}
$$

But then these two inequalities and condition (iii) in the lemma imply that

$$
C_{p_{0}, \sigma_{0 i}}\left(p_{i}^{\prime}\right)+D_{p_{0}, \sigma_{0 i}}\left(p_{i}^{\prime}\right)>C_{p_{0}, \sigma_{0 i}}\left(p_{i}\right)+D_{p_{0}, \sigma_{0 i}}\left(p_{i}\right) .
$$

But $P$ cannot be shorter than an LSP, a contradiction.

Lemma 3.4 below shows that when we slide an LSP, the path cost changes linearly.

Lemma 3.4. Let $P=\left(p_{0}, \ldots, p_{k}\right)$ be an LSP with edge sequence $\sigma$, where $p_{i}$ lies in the interior of $e_{i}$ for $i \in[1, k]$. Let $Q=\left(q_{0}, \ldots, q_{k}\right)$ be another path such that $q_{i} \in e_{i}$ for $i \in[0, k]$ and $q_{i-1} q_{i}$ is parallel to $p_{i-1} p_{i}$ for $i \in[1, k]$. For $i, j \in[0, k]$ such that $i<j$, define $\delta_{i j}$ and $\gamma_{i j}$ by the relations $\left\|p_{i} q_{i}\right\|=\delta_{i j} \cdot\left\|p_{j} q_{j}\right\|$ and $\operatorname{cost}\left(Q\left[q_{i}, q_{j}\right]\right)=\operatorname{cost}\left(P\left[p_{i}, p_{j}\right]\right)+$ $\gamma_{i j} \cdot\left\langle q_{j}-p_{j}, \hat{e_{j}}\right\rangle$. Then, $\delta_{i j}$ and $\gamma_{i j}$ depend on the direction vector of $P\left[p_{i}, p_{j}\right]$ only, $\delta_{i-1, i}$ and $\gamma_{i-1, i}$ can be computed in $O(1)$ time, and for all $\ell \in[i+1, j-1], \delta_{i j}=\delta_{i \ell} \delta_{\ell j}$ and $\gamma_{i j}=\delta_{\ell j} \gamma_{i \ell}+\gamma_{\ell j}$.

Proof. Let $\hat{v}_{i}$ be the direction of $p_{i-1} p_{i}$. By the sine law, $\delta_{i-1, i}=\sin \left(\theta\left(\hat{v}_{i}, \hat{e}_{i-1}\right)\right) / \sin \left(\theta\left(\hat{v}_{i}, \hat{e}_{i}\right)\right)$. The edges $e_{i-1}$ and $e_{i}$ share a negative endpoint $a$ or a positive endpoint $b$, and $\left\|q_{i-1} q_{i}\right\|=\sin \left(\theta\left(\hat{e}_{i}, \hat{e}_{i-1}\right)\right) \cdot\left\|a q_{i}\right\| / \sin \left(\theta\left(\hat{v}_{i}, \hat{e}_{i-1}\right)\right)$ and $\left\|q_{i-1} q_{i}\right\|=\sin \left(\theta\left(\hat{e}_{i-1}, \hat{e}_{i}\right)\right) \cdot\left\|q_{i} b\right\| / \sin \left(\theta\left(\hat{v}_{i}, \hat{e}_{i-1}\right)\right)$, respectively. Similar identities hold for $\left\|p_{i-1} p_{i}\right\|$. Thus, $\gamma_{i-1, i}=$ $c_{i} \cdot \sin \left(\theta\left(\hat{e}_{i}, \hat{e}_{i-1}\right)\right) / \sin \left(\theta\left(\hat{v}_{i}, \hat{e}_{i-1}\right)\right)$, where $c_{i}$ is the cost of a unit segment with direction $\hat{v}_{i}$ in the face bounded by $e_{i-1}$ and $e_{i}$. So $\delta_{i-1, i}$ and $\gamma_{i-1, i}$ depend on $\hat{v}_{i}$ only. Assume that $i<j-1$. For all $\ell \in(i, j),\left\|p_{i} q_{i}\right\|=\delta_{i \ell} \cdot\left\|p_{\ell} q_{\ell}\right\|=$ $\delta_{i \ell} \delta_{\ell j} \cdot\left\|p_{j} q_{j}\right\|$, and

$$
\begin{aligned}
& \operatorname{cost}\left(Q\left[q_{i}, q_{j}\right]\right) \\
= & \operatorname{cost}\left(Q\left[q_{i}, q_{\ell}\right]\right)+\operatorname{cost}\left(Q\left[q_{\ell}, q_{j}\right]\right) \\
= & \operatorname{cost}\left(P\left[p_{i}, p_{\ell}\right]\right)+\gamma_{i \ell} \cdot\left\langle q_{\ell}-p_{\ell}, \hat{e}_{\ell}\right\rangle \\
& +\operatorname{cost}\left(P\left[p_{\ell}, p_{j}\right]\right)+\gamma_{\ell j} \cdot\left\langle q_{j}-p_{j}, \hat{e}_{j}\right\rangle \\
= & \operatorname{cost}\left(P\left[p_{i}, p_{j}\right]\right)+\left(\delta_{\ell j} \gamma_{i \ell}+\gamma_{\ell j}\right) \cdot\left\langle q_{j}-p_{j}, \hat{e}_{j}\right\rangle .
\end{aligned}
$$

So $\delta_{i j}=\delta_{i \ell} \delta_{\ell j}$ and $\gamma_{i j}=\delta_{\ell j} \gamma_{i \ell}+\gamma_{\ell j}$. Inductively, $\delta_{i j}$ and $\gamma_{i j}$ depend on the direction vector of $P[i, j]$ only.

We want to show that $\partial C_{p_{0}, \sigma_{0 k}}^{+}, \partial C_{p_{0}, \sigma_{0 k}}^{-}, \partial D_{p_{k}, \sigma_{0 k}}^{+}$, and $\partial D_{p_{k}, \sigma_{0 k}}^{-}$depend on the direction vector only, i.e., not on the location of $p_{0}$ and $p_{k}$. Then, Lemmas 3.3 and 3.4 allow us to form canonical LSPs by sliding and concatenating shorter ones. The first step is a conditional version of this result.

Lemma 3.5. Let $P=\left(p_{0}, \ldots, p_{k}\right)$ be an LSP with edge sequence $\sigma$, where $p_{i}$ lies in the interior of $e_{i}$ for $i \in[1, k]$. Define $\delta_{i j}$ and $\gamma_{i j}$ as in Lemma 3.4. If there exists $i \in$ $[1, k-1]$ such that $\partial C_{p_{0}, \sigma_{0 i}}^{+}$and $\partial C_{p_{0}, \sigma_{0 i}}^{-}$depend only on the director vector of $P\left[p_{0}, p_{i}\right]$, and $\partial D_{p_{k}, \sigma_{i k}}^{+}$and $\partial D_{p_{k}, \sigma_{i k}}^{-}$ depend only on the direction vector of $P\left[p_{i}, p_{k}\right]$, then:

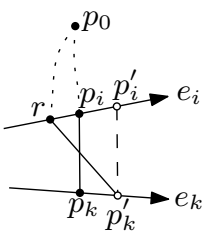

(a)

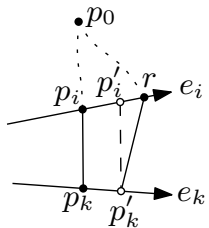

(b)

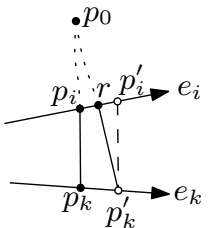

(c)
Figure 1: Three cases depending on the position of $r$ relative to $p_{i}$ and $p_{i}^{\prime}$.

(i) $\partial C_{p_{0}, \sigma_{0 k}}^{+}\left(p_{k}\right)=\min \left\{\partial C_{p_{i}, \sigma_{i k}}^{+}\left(p_{k}\right), \delta_{i k} \cdot \partial C_{p_{0}, \sigma_{0 i}}^{+}\left(p_{i}\right)+\right.$ $\left.\gamma_{i k}\right\}$ and $\partial C_{p_{0}, \sigma_{0 k}}^{-}\left(p_{k}\right)=\min \left\{\partial C_{p_{i}, \sigma_{i k}}^{-}\left(p_{k}\right), \delta_{i k} \cdot \partial C_{p_{0}, \sigma_{0 i}}^{-}\left(p_{i}\right)+\right.$ $\left.\gamma_{i k}\right\}$.

(ii) $\partial D_{p_{k}, \sigma_{0 k}}^{+}\left(p_{0}\right)=\min \left\{\partial D_{p_{i}, \sigma_{0 i}}^{+}\left(p_{0}\right), \frac{1}{\delta_{0 i}} \partial D_{p_{k}, \sigma_{i k}}^{+}\left(p_{i}\right)+\right.$ $\left.\frac{\gamma_{0 i}}{\delta_{0 i}}\right\}$ and

$\partial D_{p_{k}, \sigma_{0 k}}^{-}\left(p_{0}\right)=\min \left\{\partial D_{p_{i}, \sigma_{0 i}}^{-}\left(p_{0}\right), \frac{1}{\delta_{0 i}} \partial D_{p_{0}, \sigma_{0 i}}^{-}\left(p_{i}\right)+\right.$ $\left.\frac{\gamma_{0 i}}{\delta_{0 i}}\right\}$.

Proof. Consider the derivation of $\partial C_{p_{0}, \sigma_{0 k}}^{+}\left(p_{k}\right)$ in (i). The derivation of $\partial C_{p_{0}, \sigma_{0 k}}^{-}\left(p_{k}\right)$ is symmetric. Take a point $p_{k}^{\prime} \in e_{k}$ on the positive side of $p_{k}$ and arbitrarily close to $p_{k}$. For $j \in[i, k]$, let $p_{j}^{\prime}$ be the point in $e_{j}$ such that $p_{j}^{\prime} p_{j+1}^{\prime}$ is parallel to $p_{j} p_{j+1}$. Since $p_{k}^{\prime}$ is arbitrarily close to $p_{k}, p_{i}^{\prime}$ is also arbitrarily close to $p_{i}$. Therefore,

$$
\begin{aligned}
& C_{p_{0}, \sigma_{0 i}}\left(p_{i}\right)+C_{p_{i}, \sigma_{i k}}\left(p_{k}^{\prime}\right) \\
= & C_{p_{0}, \sigma_{0 i}}\left(p_{i}\right)+C_{p_{i}, \sigma_{i k}}\left(p_{k}\right)+\partial C_{p_{i}, \sigma_{i k}}^{+}\left(p_{k}\right) \cdot\left\|p_{k} p_{k}^{\prime}\right\| \\
= & C_{p_{0}, \sigma_{0 k}}\left(p_{k}\right)+\partial C_{p_{i}, \sigma_{i k}}^{+}\left(p_{k}\right) \cdot\left\|p_{k} p_{k}^{\prime}\right\|, \text { and } \\
& C_{p_{0}, \sigma_{0 i}}\left(p_{i}^{\prime}\right)+C_{p_{i}^{\prime}, \sigma_{i k}}\left(p_{k}^{\prime}\right) \\
= & C_{p_{0}, \sigma_{0 i}}\left(p_{i}\right)+\partial C_{p_{0}, \sigma_{0 i}}^{+}\left(p_{i}\right) \cdot\left\|p_{i} p_{i}^{\prime}\right\|+ \\
& C_{p_{i}, \sigma_{i k}}\left(p_{k}\right)+\gamma_{i k} \cdot\left\|p_{k} p_{k}^{\prime}\right\| \\
= & C_{p_{0}, \sigma_{0 k}}\left(p_{k}\right)+\left(\delta_{i k} \cdot \partial C_{p_{0}, \sigma_{0 k}}^{+}\left(p_{k}\right)+\gamma_{i k}\right) \cdot\left\|p_{k} p_{k}^{\prime}\right\| .
\end{aligned}
$$

The correctness of (i) follows if we can show that $C_{p_{0}, \sigma_{0 k}}\left(p_{k}^{\prime}\right)$ equals $C_{p_{0}, \sigma_{0 i}}\left(p_{i}\right)+C_{p_{i}, \sigma_{i k}}\left(p_{k}^{\prime}\right)$ or $C_{p_{0}, \sigma_{0 i}}\left(p_{i}^{\prime}\right)+C_{p_{i}^{\prime}, \sigma_{i k}}\left(p_{k}^{\prime}\right)$.

Let $Q$ be an LSP from $p_{0}$ to $p_{k}^{\prime}$ with edge sequence $\sigma_{0 k}=$ $\sigma$. Let $r$ be the node of $Q$ on $e_{i}$. There are three cases as shown in Figure 1 depending on the position of $r$.

Suppose that $r$ is on the negative side of $p_{i}$. See Figure 1(a). $Q\left[r, p_{k}^{\prime}\right]$ and $P\left[p_{i}, p_{k}\right]$ cross in this case, say at point $x$. Since $P$ and $Q$ are LSPs, their subpaths are also LSPs. Thus, $\operatorname{cost}\left(P\left[p_{0}, x\right]\right)=\operatorname{cost}\left(Q\left[p_{0}, x\right]\right)$, and so $C_{p_{0}, \sigma_{0 k}}\left(p_{k}^{\prime}\right)=$ $\operatorname{cost}(Q)=\operatorname{cost}\left(P\left[p_{0}, x\right]\right)+\operatorname{cost}\left(Q\left[x, p_{k}^{\prime}\right]\right) \geq C_{p_{0}, \sigma_{0 i}}\left(p_{i}\right)+$ $C_{p_{i}, \sigma_{i k}}\left(p_{k}^{\prime}\right)$. An LSP to $p_{k}^{\prime}$ cannot cost more than any path to $p_{k}^{\prime}$ via $p_{i}$. Thus, $C_{p_{0}, \sigma_{0 k}}\left(p_{k}^{\prime}\right)=C_{p_{0}, \sigma_{0 i}}\left(p_{i}\right)+C_{p_{i}, \sigma_{i k}}\left(p_{k}^{\prime}\right)$.

Suppose that $r$ is on the positive side of $p_{i}^{\prime}$. See Figure 1(b). Since $C_{p_{0}, \sigma_{0 i}}$ is a convex function by Lemma 3.2, $C_{p_{0}, \sigma_{0 i}}(r) \geq C_{p_{0}, \sigma_{0 i}}\left(p_{i}\right)+\partial C_{p_{0}, \sigma_{0 i}}^{+}\left(p_{i}\right) \cdot\left\|p_{i} r\right\|=C_{p_{0}, \sigma_{0 i}}\left(p_{i}^{\prime}\right)+$ $\partial C_{p_{0}, \sigma_{0 i}}^{+}\left(p_{i}\right) \cdot\left\|p_{i}^{\prime} r\right\|$, where the last equality follows from the fact that $p_{i}^{\prime}$ is arbitrarily close to $p_{i}$. Because $p_{j}^{\prime} p_{j+1}^{\prime}$ is parallel to $p_{j} p_{j+1}$ for all $j \in[i, k-1]$, we obtain $\partial D_{p_{k}^{\prime}, \sigma_{i k}}^{+}\left(p_{i}^{\prime}\right)=$ $\partial D_{p_{k}, \sigma_{i k}}^{+}\left(p_{i}\right)$ by the assumption that $\partial D_{p_{k}, \sigma_{i k}}^{+}$depends on the direction vector of $P[i, j]$ only. Thus, $D_{p_{k}^{\prime}, \sigma_{i k}}(r) \geq$ $D_{p_{k}^{\prime}, \sigma_{i k}}\left(p_{i}^{\prime}\right)+\partial D_{p_{k}^{\prime}, \sigma_{i k}}^{+}\left(p_{i}^{\prime}\right) \cdot\left\|p_{i}^{\prime} r\right\|=C_{p_{i}^{\prime}, \sigma_{i k}}\left(p_{k}^{\prime}\right)+\partial D_{p_{k}, \sigma_{i k}}^{+}\left(p_{i}\right)$. $\left\|p_{i}^{\prime} r\right\|$. By combining the two inequalities above, we obtain $C_{p_{0}, \sigma_{0 k}}\left(p_{k}^{\prime}\right)=C_{p_{0}, \sigma_{0 i}}(r)+D_{p_{k}^{\prime}, \sigma_{i k}}(r) \geq C_{p_{0}, \sigma_{0 i}}\left(p_{i}^{\prime}\right)+$ 

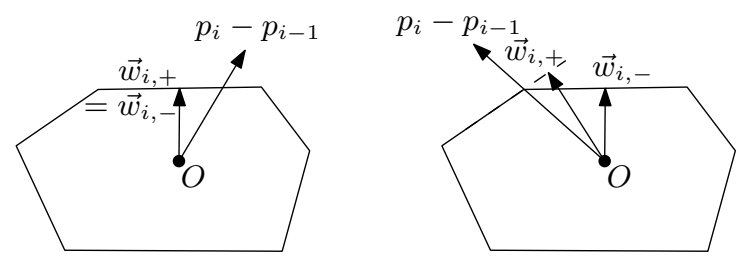

Figure 2: Left: The ray in the direction of $p_{i}-p_{i-1}$ crosses the boundary of $H_{f_{i}}$ at a point that is not a vertex. $\vec{w}_{i,-}=\vec{w}_{i,+}$. Right: The ray in the direction of $p_{i}-p_{i-1}$ crosses the boundary of $H_{f_{i}}$ at a vertex, so $\vec{w}_{i,-}$ and $\vec{w}_{i,+}$ are defined by the edges of $H_{f_{i}}$ incident to that vertex.

$C_{p_{i}^{\prime} \sigma_{i k}}\left(p_{k}^{\prime}\right)+\left(\partial C_{p_{0}, \sigma_{i k}}^{+}\left(p_{i}\right)+\partial D_{p_{k}, \sigma_{i k}}^{+}\left(p_{i}\right)\right)\left\|p_{i}^{\prime} r\right\|$, which is at least $C_{p_{0}, \sigma_{0 i}}\left(p_{i}^{\prime}\right)+C_{p_{i}^{\prime} \sigma_{i k}}\left(p_{k}^{\prime}\right)$ by Lemma 3.3.

Suppose that $r \in p_{i} p_{i}^{\prime}$. See Figure 1(c). Since $\left\|p_{i} r\right\|$ and $\left\|r p_{i}^{\prime}\right\|$ are arbitrarily small, $C_{p_{0}, \sigma_{0 k}}\left(p_{k}^{\prime}\right)=C_{p_{0}, \sigma_{0 i}}(r)+$ $D_{p_{k}^{\prime}, \sigma_{i k}}(r)=C_{p_{0}, \sigma_{0 i}}\left(p_{i}\right)+\partial C_{p_{0}, \sigma_{0 i}}^{+}\left(p_{i}\right) \cdot\left\|p_{i} r\right\|+D_{p_{k}^{\prime}, \sigma_{i k}}\left(p_{i}^{\prime}\right)-$ $\partial D_{p_{k}^{\prime}, \sigma_{i k}}^{+}\left(p_{i}^{\prime}\right) \cdot\left\|p_{i}^{\prime} r\right\|$, which is linear in $\left\|p_{i} r\right\|$ by our assumption that $\partial C_{p_{0}, \sigma_{0 i}}^{+}$and $\partial D_{p_{k}, \sigma_{i k}}^{+}$depend only on the direction vectors of $P\left[p_{0}, p_{i}\right]$ and $P[i, k]$ (hence $\partial D_{p_{k}^{\prime}, \sigma_{i k}}^{+}\left(p_{i}^{\prime}\right)=$ $\left.\partial D_{p_{k}, \sigma_{i k}}^{+}\left(p_{i}\right)\right)$. Thus, $C_{p_{0}, \sigma_{0 k}}\left(p_{k}^{\prime}\right)$ is minimized when $r=$ $p_{i}$ or $r=p_{i}^{\prime}$. which means $C_{p_{0}, \sigma_{0 k}}\left(p_{k}^{\prime}\right)=C_{p_{0}, \sigma_{0 i}}\left(p_{i}\right)+$ $D_{p_{k}^{\prime}, \sigma_{i k}}\left(p_{i}\right)=C_{p_{0}, \sigma_{0 i}}\left(p_{i}\right)+C_{p_{i}, \sigma_{i k}}\left(p_{k}^{\prime}\right)$ or $C_{p_{0}, \sigma_{0 k}}\left(p_{k}^{\prime}\right)=$ $C_{p_{0}, \sigma_{0 i}}\left(p_{i}^{\prime}\right)+D_{p_{k}^{\prime}, \sigma_{i k}}\left(p_{i}^{\prime}\right)=C_{p_{0}, \sigma_{0 i}}\left(p_{i}^{\prime}\right)+C_{p_{i}^{\prime}, \sigma_{i k}}\left(p_{k}^{\prime}\right)$.

The correctness of (ii) can be proved in a similar way.

Lemma 3.5 lends itself to an inductive proof to establish the same result unconditionally, as stated in Lemma 3.6.

Lemma 3.6. Let $P=\left(p_{0}, \ldots, p_{k}\right)$ be an LSP with edge sequence $\sigma$, where $p_{i}$ lies in the interior of $e_{i}$ for $i \in[1, k]$. Let $\delta_{i j}$ and $\gamma_{i j}$ be defined as in Lemma 3.4. Then $\partial C_{p_{0}, \sigma_{0 k}}^{+}$, $\partial C_{p_{0}, \sigma_{0 k}}^{-}, \partial D_{p_{k}, \sigma_{0 k}}^{+}$, and $\partial D_{p_{k}, \sigma_{0 k}}^{-}$depend only on the direction vector of P. Moreover, the formulae in Lemma 3.5 hold for all $i \in[1, k-1]$.

Proof. We first show that $\partial C_{p_{i-1},\left(e_{i}\right)}^{+}\left(p_{i}\right)$ depends only on the direction of $p_{i}-p_{i-1}$. Divide all directions into cones, each being the set of directions from the origin to all points in one edge of the polygon $H_{f_{i}}$ defining the distance function for the face $f$ bound by $e_{i-1}$ and $e_{i}$.

If $p_{i}-p_{i-1}$ points to a vertex of $H_{f_{i}}$, there are two cones that contain $p_{i}-p_{i-1}$. We use $\ell_{-}$to denote the support line of the edge of $H_{f_{i}}$ defining the cone that comes first in anticlockwise order among these two cones, and $\ell_{+}$denotes the support line of the edge of $H_{f_{i}}$ that defines the other cone. If $p_{i}-p_{i-1}$ points to the interior of an edge of $H_{f_{i}}$, then both $\ell_{+}$and $\ell_{-}$denote the support line of this edge. Let $\vec{w}_{i,+}$ and $\vec{w}_{i,-}$ be the vectors that are orthogonal to $\ell_{+}$and $\ell_{-}$, respectively. See Figure 2 . It follows that $\operatorname{cost}\left(p_{i-1} p_{i}^{\prime}\right)=\frac{\left\langle p_{i}^{\prime}-p_{i-1}, \vec{w}_{i,+}\right\rangle}{\left\|\vec{w}_{i,+}\right\|^{2}}$ and $\operatorname{cost}\left(p_{i-1} p_{i}\right)=$ $\frac{\left\langle p_{i}-p_{i-1}, \vec{w}_{i,+}\right\rangle}{\left\|\vec{w}_{i,+}\right\|^{2}}$. So $\partial C_{p_{i-1},\left(e_{i}\right)}^{+}\left(p_{i}\right)=\frac{\left\langle\hat{e}_{i}, \vec{w}_{i,+}\right\rangle}{\left\|\vec{w}_{i,+}\right\|^{2}}$, which only depends on the direction of $p_{i}-p_{i-1}$. Similarly, one can verify that $\partial C_{p_{i-1},\left(e_{i}\right)}^{-}\left(p_{i}\right)=\frac{\left\langle\hat{e}_{i}, \vec{w}_{i,-}\right\rangle}{\left\|\vec{w}_{i,-}\right\|^{2}}, \partial D_{p_{i},\left(e_{i}\right)}^{+}\left(p_{i-1}\right)=$ $-\frac{\left\langle\hat{e}_{i-1}, \vec{w}_{i,+}\right\rangle}{\left\|\vec{w}_{i,+}\right\|^{2}}, \partial D_{p_{i},\left(e_{i}\right)}^{-}\left(p_{i-1}\right)=-\frac{\left\langle\hat{e}_{i-1}, \vec{w}_{i,-}\right\rangle}{\left\|\vec{w}_{i,-}\right\|^{2}}$. They all depend on the direction of $p_{i}-p_{i-1}$ only.

$\partial C_{p_{0}, \sigma_{01}}^{+}\left(p_{1}\right)$ and $\partial C_{p_{0}, \sigma_{01}}^{-}\left(p_{1}\right)$ depend only on the direction of $p_{1}-p_{0}$ as discussed above. Applying Lemma 3.5(i)
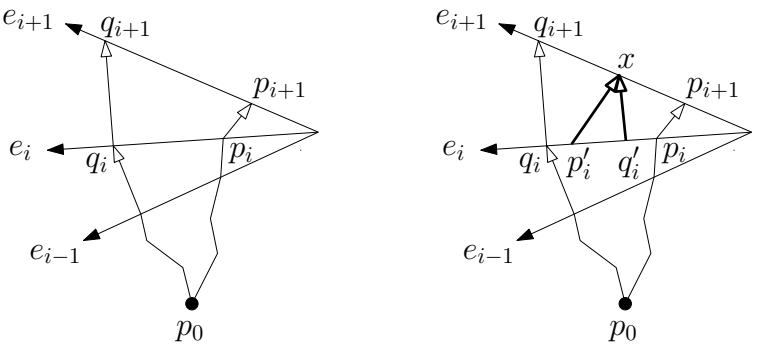

Figure 3: Illustration for the proof of Lemma 3.7.

with $i=1$ and $k=2$ shows that $\partial C_{p_{0}, \sigma_{02}}^{+}\left(p_{2}\right)$ and $\partial C_{p_{0}, \sigma_{02}}^{-}\left(p_{2}\right)$ depend only on the directions of $p_{1}-p_{0}$ and $p_{2}-p_{1}$. By repeatedly applying Lemma $3.5(\mathrm{i})$ with $k=i+1$, one can show that $\partial C_{p_{0}, \sigma_{0 i}}^{+}\left(p_{i}\right)$ and $\partial C_{p_{0}, \sigma_{0 i}}^{-}\left(p_{i}\right)$ depend only on the direction vector of $P\left[p_{0}, p_{i}\right]$ for all $i \in[1, k-1]$.

By Lemma 3.5(ii), one can similarly show that $\partial D_{p_{k}, \sigma_{i k}}^{+}\left(p_{i}\right)$ and $\partial D_{p_{k}, \sigma_{i k}}^{-}\left(p_{i}\right)$ depend only on the direction vector of $P\left[p_{i}, p_{k}\right]$. Thus, the conditions on Lemma 3.5(i) and (ii) can be removed.

Lemma 3.7 below follows from Lemmas 3.2, 3.3, and 3.6. It implies that once two canonical LSPs diverge, they cannot cross afterward.

Lemma 3.7. Let $P=\left(p_{0}, \ldots, p_{k}\right)$ and $Q=\left(q_{0}, \ldots, q_{k}\right)$ be two canonical LSPs with edge sequence $\sigma$ such that $p_{i}$ and $q_{i}$ lie in the interior of $e_{i}$ for $i \in[1, k-1]$. If $\theta\left(p_{i}-p_{i-1}, \vec{e}_{i}\right)>$ $\theta\left(q_{i}-q_{i-1}, \vec{e}_{i}\right)$ for some $i \in[1, k-1]$, then $\theta\left(p_{j}-p_{j-1}, \vec{e}_{j}\right) \geq$ $\theta\left(q_{j}-q_{j-1}, \vec{e}_{j}\right)$ for all $j>i$.

Proof. It suffices to show that if $\theta\left(p_{\ell}-p_{\ell-1}, \vec{e}_{\ell}\right) \geq \theta\left(q_{\ell}-\right.$ $\left.q_{\ell-1}, \vec{e}_{\ell}\right)$ for all $\ell \in[1, i]$ and $\theta\left(p_{\ell}-p_{\ell-1}, \vec{e}_{\ell}\right)>\theta\left(q_{\ell}-\bar{q}_{\ell-1}, \vec{e}_{\ell}\right)$ for some $\ell \in[1, i]$, then $\theta\left(p_{i+1}-p_{i}, \vec{e}_{i+1}\right) \geq \theta\left(q_{i+1}-q_{i}, \vec{e}_{i+1}\right)$. By Lemma 3.6, we can assume that $p_{0}=q_{0}$. So $q_{i}$ is on the positive side of $p_{i}$. Assume that $p_{i} p_{i+1}$ and $q_{i} q_{i+1}$ are not parallel because we are done otherwise.

Translate the segments $p_{i} p_{i+1}$ and $q_{i} q_{i+1}$ to obtain parallel segments $p_{i}^{\prime} x$ and $q_{i}^{\prime} x$, respectively, that meet at some point $x \in e_{i+1}$. Refer to Figure 3 . The choices of $p_{i}^{\prime}, q_{i}^{\prime}$ and $x$ are quite arbitrary as long as $p_{i} p_{i+1}$ and $q_{i} q_{i+1}$ are parallel to $p_{i}^{\prime} x$ and $q_{i}^{\prime} x$, respectively.

We claim that $\partial D_{x,\left(e_{i+1}\right)}^{+}\left(q_{i}^{\prime}\right)<\partial D_{x,\left(e_{i+1}\right)}^{+}\left(p_{i}^{\prime}\right)$. Suppose not. Then, $\partial D_{x,\left(e_{i+1}\right)}^{+}\left(q_{i}^{\prime}\right) \geq \partial D_{x,\left(e_{i+1}\right)}^{-}\left(q_{i}^{\prime}\right) \geq \partial D_{x,\left(e_{i+1}\right)}^{+}\left(p_{i}^{\prime}\right)$ as $D_{x,\left(e_{i+1}\right)}$ is convex and $p_{i}^{\prime} \neq q_{i}^{\prime}$. Lemma 3.6 implies that $\partial D_{q_{i+1},\left(e_{i+1}\right)}^{-}\left(q_{i}\right)=\partial D_{x,\left(e_{i+1}\right)}^{-}\left(q_{i}^{\prime}\right) \geq \partial D_{x,\left(e_{i+1}\right)}^{+}\left(p_{i}^{\prime}\right)=$ $\partial D_{p_{i+1},\left(e_{i+1}\right)}^{+}\left(p_{i}\right)$. Since $q_{i}$ is on the positive side of $p_{i}$, by Lemma $3.2, \partial C_{p_{0}, \sigma_{0 i}}^{-}\left(q_{i}\right) \geq \partial C_{p_{0}, \sigma_{0 i}}^{+}\left(p_{i}\right)$. Then $\partial C_{p_{0}, \sigma_{0 i}}^{-}\left(q_{i}\right)+$ $\partial D_{q_{i+1},\left(e_{i+1}\right)}^{-}\left(q_{i}\right) \geq \partial C_{p_{0}, \sigma_{0 i}}^{+}\left(p_{i}\right)+\partial D_{p_{i+1},\left(e_{i+1}\right)}^{+}\left(p_{i}\right)$, which is non-negative by Lemma 3.3(ii). This is a contradiction because $\partial C_{p_{0}, \sigma_{0 i}}^{-}\left(q_{i}\right)+\partial D_{q_{i+1},\left(e_{i+1}\right)}^{-}\left(q_{i}\right)$ should be negative by Lemma 3.3(iii).

By our claim, $q_{i}^{\prime}$ lies on the negative side of $p_{i}^{\prime}$. Since $q_{i}$ is on the positive side of $p_{i}$, the relation $\theta\left(p_{i+1}-p_{i}, \vec{e}_{i+1}\right) \geq$ $\theta\left(q_{i+1}-q_{i}, \vec{e}_{i+1}\right)$ must hold in order that the sliding switches the order of $p_{i}$ and $q_{i}$ to align $p_{i+1}$ and $q_{i+1}$.

\subsection{Algorithm}

Chen and Han introduced the sequence tree to capture the edge sequences of LSPs in the $L_{2}$ case [6]. The tree is grown 
until the number of tree levels meets the input upper bound on the number of links allowed in the solution path. The best path discovered from $s$ to $t$ is the shortest path desired. Constructing a new tree node involves finding a new shortest path with a particular edge sequence. The key is to use the structural properties in the last subsection to carry out this step and do it fast.

A sequence tree node $\alpha$ is a vertex-node or an edge-node which represents a vertex, denoted $\nu_{\alpha}$, or an edge of $\mathcal{T}$, denoted $e_{\alpha}$. A face corner $(f, \nu)$ is the corner at a vertex $\nu$ of a face $f$. An edge-node $\alpha$ annexes a face corner $(f, \nu)$ if $e_{\alpha}$ is the edge of $f$ opposite $\nu$ and the parent of $\alpha$ does not correspond to another vertex or edge of $f$. (Since $e_{\alpha}$ is opposite two face corners, the second condition ensures that $\alpha$ annexes the face corner just included by the growing tree.)

The root corresponds to the source $s$. The nodes on the tree path from the root to $\alpha$ correspond to an edge sequence, denoted $\sigma_{\alpha}$. Let $\alpha_{0}$ be the nearest ancestor vertex-node of $\alpha$. The edge-nodes on the tree path from $\alpha_{0}$ to $\alpha$ correspond to a suffix of $\sigma_{\alpha}$, denoted $\tilde{\sigma}_{\alpha}$. The edge sequences $\sigma_{\alpha}$ and $\tilde{\sigma}_{\alpha}$ are used in the analysis, but they are not stored at $\alpha$. If $\alpha$ is a vertex-node, $P_{\alpha}$ denotes the canonical LSP from $s$ to $\nu_{\alpha}$ that passes through the edges in $\sigma_{\alpha}$. We compute $\operatorname{cost}\left(P_{\alpha}\right)$ and store it at $\alpha$, but $P_{\alpha}$ is used in the analysis only.

The sequence tree is grown in a breadth-first manner until the number of tree levels meets the input upper bound $m$. When an edge-node annexing a face corner $(f, \nu)$ is expanded, it gains at most one vertex-node corresponding to $\nu$ and two edge-nodes corresponding to the edges of $f$ incident to $\nu$. When a vertex-node $\alpha$ is expanded, it gains at most one vertex-node for each vertex adjacent to $\nu_{\alpha}$ and one edge-node for each edge opposite $\nu_{\alpha}$. Multiple nodes may correspond to the same edge or vertex. To control the tree size, Chan and Han introduced the one-corner one-split property: at any time, at most one vertex-node corresponding to the same vertex is allowed to have any child node; at most one edge-node annexing the same face corner is allowed to have two child edge-nodes. This property ensures that at most $O(n)$ tree nodes are ever created at each level [6, Theorem 8]. This is the reason why we forbid critical refractions. If they are allowed, the one-corner one-split property cannot be enforced and the sequence tree may be much larger.

A notion of dominance is needed to maintain the onecorner one-split property. Let $\alpha$ and $\beta$ be two vertex-nodes corresponding to the same vertex $\nu$ or two edge-nodes annexing the same face corner $(f, \nu)$. Let $\alpha_{0}$ and $\beta_{0}$ be the nearest ancestor vertex-nodes of $\alpha$ and $\beta$, respectively. Let $P$ and $Q$ be the canonical LSPs from $\nu_{\alpha_{0}}$ and $\nu_{\beta_{0}}$ to $\nu$ that pass through the edges in $\tilde{\sigma}_{\alpha}$ and $\tilde{\sigma}_{\beta}$, respectively. We say that $\alpha$ dominates $\beta$ if $\operatorname{cost}\left(P_{\alpha_{0}}\right)+\operatorname{cost}(P)<\operatorname{cost}\left(P_{\beta_{0}}\right)+\operatorname{cost}(Q)$, or $\operatorname{cost}\left(P_{\alpha_{0}}\right)+\operatorname{cost}(P)=\operatorname{cost}\left(P_{\beta_{0}}\right)+\operatorname{cost}(Q)$ but $\alpha$ is expanded before $\beta$ in growing the tree. Assume that $\alpha$ dominates $\beta$. Suppose they are vertex-nodes. If $\beta$ has been expanded, we remove all tree nodes descending from it; otherwise, we will not expand $\beta$. Suppose that $\alpha$ and $\beta$ are edge-nodes. There is an edge $e$ incident to $\nu$ such that every LSP from $\nu_{\beta_{0}}$ to $e$ through the edges in $\tilde{\sigma}_{\beta}$ crosses $P$. If $\beta$ has been expanded, we prune the child node of $\beta$ corresponding to $e$; otherwise, when we expand $\beta$, we will not generate a child node corresponding to $e$.

After we construct a new leaf $\alpha$ of the sequence tree, it takes $O(\log m n)$ amortized time to test the dominance and prune the tree, modulo the time to compute the costs of
LSPs: $\operatorname{cost}\left(P_{\alpha}\right)$ if $\alpha$ is a vertex-node, or the costs of LSPs from $\nu_{\alpha_{0}}$ to $e_{\alpha}$ with edge sequence $\tilde{\sigma}_{\alpha}$ if $\alpha$ is an edge-node. In the rest of this subsection, we describe the dominance testing, the pruning, and the computation of the costs of LSPs when constructing a new leaf.

\subsubsection{Dominance checking and tree pruning}

The vertex-node case is easy. For each vertex of $\mathcal{T}$, we record the current corresponding vertex-node $\beta$ that dominates all other vertex-nodes corresponding to $\nu_{\beta}$. When a new vertex-node $\alpha$ corresponding to $\nu_{\beta}$ is created, we compare $\alpha$ and $\beta$ to see which of the two dominates the other. If $\beta$ is dominated, we delete all descendants of $\beta$. A node can only be deleted at most once. We charge the pruning work to the creation of the pruned nodes. Thus, it takes only $O(1)$ amortized time modulo the time for computing the cost of the LSP from $s$ to $\nu_{\alpha}$ with edge sequence $\sigma_{\alpha}$.

It takes more time to handle edge-nodes. For every face corner $(f, \nu)$, we record the edge-node $\beta$ that annexes $(f, \nu)$ and dominates all other edge-nodes annexing $(f, \nu)$. We say that $\beta$ occupies $(f, \nu)$. Suppose that a new edge-node $\alpha$ annexing $(f, \nu)$ is generated. Let $\alpha^{\prime}$ and $\beta^{\prime}$ be the nearest proper ancestor vertex-nodes of $\alpha$ and $\beta$, respectively. Let $P$ and $Q$ be the LSPs from $\nu_{\alpha^{\prime}}$ and $\nu_{\beta^{\prime}}$ to $\nu$ through the edges in $\tilde{\sigma}_{\sigma}$ and $\tilde{\sigma}_{\beta}$, respectively. We must have computed and recorded $\operatorname{cost}\left(P_{\beta^{\prime}}\right)+\operatorname{cost}(Q)$ beforehand as $\beta$ occupies $(f, \nu)$. Therefore, modulo the time to compute $\operatorname{cost}(P)$, we can compare $\operatorname{cost}\left(P_{\alpha^{\prime}}\right)+\operatorname{cost}(P)$ with $\operatorname{cost}\left(P_{\beta^{\prime}}\right)+\operatorname{cost}(Q)$ to decide the dominance in $O(1)$ time. Without loss of generality, assume that $\alpha$ dominates $\beta$. Then, $\alpha$ replaces $\beta$ as the edge-node that occupies $(f, \nu)$.

To decide which child edge-node of $\beta$ to prune, we need to refine the notion of dominance. Consider the two edge sequences $\sigma_{\alpha}$ and $\sigma_{\beta}$. Let $e$ denote the first edge in the longest common suffix of $\sigma_{\alpha}$ and $\sigma_{\beta}$.

- If $\sigma_{\alpha}$ is not a suffix of $\sigma_{\beta}$, let $e_{\alpha}$ be the edge in $\sigma_{\alpha}$ before $e$. Then $\alpha$ dominates $\beta$ on the positive side (resp. negative side) if $e_{\alpha}$ and $e$ share the positive (resp. negative) endpoint. Refer to Figure 4(top).

- If $\sigma_{\alpha}$ is a suffix of $\sigma_{\beta}$, let $e_{\beta}$ be the edge in $\sigma_{\beta}$ before $e$, and $\alpha$ dominates $\beta$ on the positive side (resp. negative side) if $e_{\beta}$ and $e$ share the negative (resp. positive) endpoint. Refer to Figure 4(bottom).

We use $e^{+}$and $e^{-}$to denote the two edges of $f$ incident to $\nu$ such that $\nu$ is the negative and positive endpoints of $e^{+}$and $e^{-}$, respectively. Suppose that $\alpha$ dominates $\beta$ on the positive side. If $\beta$ has been expanded, we delete the child node of $\beta$ corresponding to $e^{+}$as well as its descendants. Again, this pruning takes $O(1)$ amortized time. If $\beta$ has not yet been expanded, we will not let $\beta$ gain a child node corresponding to $e^{+}$. The pruning is symmetric if $\alpha$ dominates $\beta$ on the negative side.

Tracing $\sigma_{\alpha}$ and $\sigma_{\beta}$ to decide whether $\alpha$ dominates $\beta$ on the positive or negative side would take $\Theta\left(\min \left\{\left|\sigma_{\alpha}\right|,\left|\sigma_{\beta}\right|\right\}\right)$ time. Instead, we use some data structures for making this decision. For every face corner $(f, \nu)$, we maintain an ordered list of edge-nodes annexing it. These edge-nodes correspond to the same edge $e$ of $f$. Let $u^{+}$and $u^{-}$be the positive and negative endpoints of $e$, respectively. Let $g=w u^{+} u^{-}$be the face of $\mathcal{T}$ that shares $e$ with $f$. The ordering of two edgenodes $\alpha$ and $\beta$ in the ordered list for $(f, \nu)$ is determined 

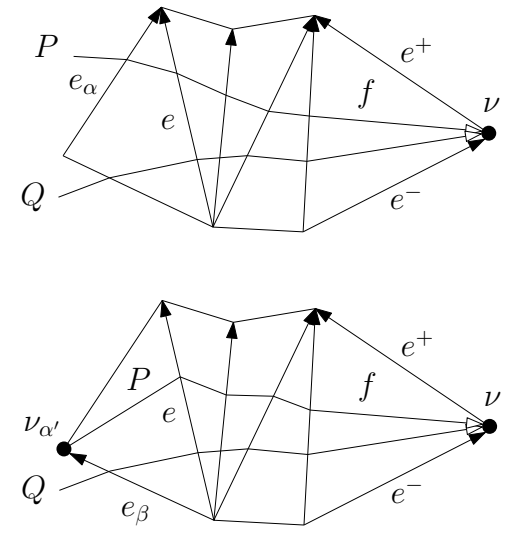

Figure 4: $\alpha$ dominates $\beta$ on the positive side. The child-node of $\beta$ corresponding to $e^{+}$will have no descendant in the sequence tree.

as follows. Let $\alpha^{\prime}$ and $\beta^{\prime}$ be the parent nodes of $\alpha$ and $\beta$, respectively.

- Suppose that $\alpha^{\prime}$ and $\beta^{\prime}$ are edge-nodes annexing different corners of $g$. If $e_{\alpha^{\prime}}$ and $e$ share the common positive endpoint $u^{+}$, then $\alpha$ precedes $\beta$ in the ordered list for $(f, \nu)$; otherwise, $\beta$ precedes $\alpha$.

- If $\alpha^{\prime}$ and $\beta^{\prime}$ are edge-nodes annexing the same corner of $g$, and $\alpha^{\prime}$ precedes $\beta^{\prime}$ in the ordered list for that face corner, then $\alpha$ precedes $\beta$ in the ordered list for $(f, \nu)$.

- If $\beta^{\prime}$ is an edge-node annexing $\left(g, u^{+}\right)$and $\alpha^{\prime}$ is a vertex-node corresponding to $w$, then $\alpha$ precedes $\beta$ in the ordered list for $(f, \nu)$.

- If $\beta^{\prime}$ is a vertex-node corresponding to $w$ and $\alpha^{\prime}$ is an edge-node annexing $\left(g, u^{-}\right)$, then $\alpha$ precedes $\beta$ in the ordered list for $(f, \nu)$.

Assume that $\alpha$ dominates $\beta$. If $\alpha$ precedes $\beta$ in the ordered list for $(f, \nu)$, then $\alpha$ dominates $\beta$ on the positive side; otherwise, $\alpha$ dominates $\beta$ on the negative side. The rules above are based on the information at the parents of $\alpha$ and $\beta$ in such a way that the decision process is equivalent to tracing $\sigma_{\alpha}$ and $\sigma_{\beta}$. This explains the correctness. Since an edgenode annexing $(f, \nu)$ can change, we need to represent the sorted list for $(f, \nu)$ with a balanced binary search tree. The total size of such sorted lists is at most the sequence tree size which is $O(m n)$. Therefore, the dominance testing can be done in $O(\log m n)$ time.

The following lemma was originally proved for $L_{\infty}$ metric. Since the proof only uses the triangle inequality, the result can be generalized to our case.

Lemma 3.8 ([7, Lemma 3.1]). Let $\alpha$ and $\beta$ be two edgenodes annexing the same face corner $(f, \nu)$ such that $\alpha$ dominates $\beta$ on the positive side (resp. negative side). Let $e$ be the edge in $f$ whose negative (resp. positive) endpoint is $\nu$.

(i) $\alpha$ is not a descendant of $\beta$.

(ii) Let $\alpha_{0}$ and $\beta_{0}$ be the nearest proper ancestor vertexnodes of $\alpha$ and $\beta$, respectively. For every point $x \in e$ and every $L S P Q$ with edge sequence $\tilde{\sigma}_{\beta} \cdot(e)$ from $\nu_{\beta_{0}}$ to $\nu$, the LSP P with edge sequence $\tilde{\sigma}_{\alpha} \cdot(e)$ from $\nu_{\alpha_{0}}$ to $\nu$ satisfies $\operatorname{cost}\left(P_{\alpha_{0}}\right)+\operatorname{cost}(P) \leq \operatorname{cost}\left(P_{\beta_{0}}\right)+\operatorname{cost}(Q)$, and if they are equal, then $\alpha$ is expanded before $\beta$.

\subsubsection{Edge-node creation}

Let $\alpha$ be a new edge-node created at tree level $\ell$. Let $\alpha_{0}$ be the nearest ancestor vertex-node of $\alpha$ at tree level $\ell_{0}<\ell$. For $j \in\left(\ell_{0}, \ell\right]$, let $e_{j}$ be the edge corresponding to the edgenode at tree level $j$ on the tree path from $\alpha_{0}$ to $\alpha$. Let $e_{\ell_{0}}$ denote the edge incident to $\nu_{\alpha_{0}}$ and adjacent to $e_{\ell_{0}+1}$ in $\sigma_{\alpha}$. We do some processing at $\alpha$ to aid the future growth of the subtree rooted at $\alpha$. For all $i \geq 0$ such that $2^{i}$ divides $\ell$, we compute a data structure $\mathcal{L}_{\alpha}^{i}$ to represent the canonical LSPs from any point in $e_{\ell-2^{i}}$ to some point in $e_{\ell}$, which can be represented by their direction vectors by Lemmas 3.3 and 3.6. The insight is that only some critical direction vectors matter, and the rest can be linearly interpolated from them.

Let $I_{\alpha, \mathbf{v}} \subseteq e_{\ell-2^{i}}$ be the interval of origins of canonical LSPs that reach $e_{\ell}$ with direction vector $\mathbf{v}$ and edge sequence $\left(e_{\ell-2^{i}+1}, \ldots, e_{\ell}\right) .^{3}$ Let $A_{\alpha, \mathbf{v}}: I_{\alpha, \mathbf{v}} \rightarrow \mathbb{R}$ and $a_{\alpha, \mathbf{v}}: I_{\alpha, \mathbf{v}} \rightarrow e_{\ell}$ be functions such that $A_{\alpha, \mathbf{v}}(p)$ is the cost of the canonical LSP from $p$ to $e_{\ell}$ with direction vector $\mathbf{v}$ and edge sequence $\left(e_{\ell-2^{i}+1}, \ldots, e_{\ell}\right)$, and $a_{\alpha, \mathbf{v}}(p)$ is the destination of this LSP. Let $B_{\alpha, \mathbf{v}}: a_{\alpha, \mathbf{v}}\left[I_{\alpha, \mathbf{v}}\right] \rightarrow \mathbb{R}$ and $b_{\alpha, \mathbf{v}}: a_{\alpha, \mathbf{v}}\left[I_{\alpha, \mathbf{v}}\right] \rightarrow e_{\ell-2^{i}}$ be functions such that $B_{\alpha, \mathbf{v}}(q)$ is the cost of the canonical LSP from $e_{\ell-2^{i}}$ to $q$ with direction vector $\mathbf{v}$ and edge sequence $\left(e_{\ell-2^{i}+1}, \ldots, e_{\ell}\right)$, and $b_{\alpha, \mathbf{v}}(q)$ is the source of this LSP. These four functions are affine and they can be stored in $O(1)$ space and evaluated in $O(1)$ time. The direction vectors in $\mathcal{L}_{\alpha}^{i}$ are stored in lexicographic order: two directions $\hat{v}_{i}$ and $\hat{w}_{i}$ for the links hitting $e_{i}$ are ordered by comparing $\theta\left(\hat{e}_{i}, \hat{v}_{i}\right)$ and $\theta\left(\hat{e}_{i}, \hat{w}_{i}\right)$. The following properties are enforced on $\mathcal{L}_{\alpha}^{i}$.

P1: Each direction vector in $\mathcal{L}_{\alpha}^{i}$ is the direction vector of some canonical LSP from $e_{\ell-2^{i}}$ to $e_{\ell}$.

P2: Any two adjacent direction vectors differ in exactly one entry. These two different directions point to the same edge of the convex polygon defining the distance function for the corresponding face.

P3: Let $\mathbf{v}$ and $\mathbf{w}$ be two adjacent direction vectors. For every $p \in I_{\alpha, \mathbf{v}} \cap I_{\alpha, \mathbf{w}}$ and every $t \in[0,1]$, the cost of an LSP from $p$ to $t a_{\alpha, \mathbf{v}}(p)+(1-t) a_{\alpha, \mathbf{w}}(p)$ is $t A_{\alpha, \mathbf{v}}(p)+$ $(1-t) A_{\alpha, \mathbf{w}}(p){ }^{4}$

$\mathrm{P} 4$ : Let $\mathbf{v}$ and $\mathbf{w}$ be two adjacent direction vectors. For every $q \in a_{\alpha, \mathbf{v}}\left[I_{\alpha, \mathbf{v}}\right] \cap a_{\alpha, \mathbf{w}}\left[I_{\alpha, \mathbf{w}}\right]$ and every $t \in[0,1]$, the cost of an LSP from $t b_{\alpha, \mathbf{v}}(q)+(1-t) b_{\alpha, \mathbf{w}}(q)$ to $q$ is $t B_{\alpha, \mathbf{v}}(q)+(1-t) B_{\alpha, \mathbf{w}}(q)$.

The first direction vector in $\mathcal{L}_{\alpha}^{i}$ is stored in its full form. For any other direction vector, we only store the directions of the first and last links and the difference from its predecessor in $\mathcal{L}_{\alpha}^{i}$. By P2, the storage required by $\mathcal{L}_{\alpha}^{i}$ is $O\left(2^{i}\right)$ plus the number of direction vectors in the list.

${ }^{3}$ Let $P$ be the canonical LSP from $I_{\alpha, \mathbf{v}}$ to $e_{\ell}$ with direction vector $\mathbf{v}$ and edge sequence $\left(e_{\ell-2^{i}+1}, \ldots, e_{\ell}\right)$. By Lemma 3.6, we can slide $P$ until it is stuck, and the path remains a canonical LSP during the sliding. Thus, $I_{\alpha, \mathbf{v}}$ is an interval, and so is $a_{\alpha, \mathbf{v}}\left[I_{\alpha, \mathbf{v}}\right]$.

${ }^{4}$ By Lemma 3.3, given two canonical LSPs from $p$ to $e_{\ell}$ with adjacent direction vectors $\mathbf{v}$ and $\mathbf{w}$, any linearly interpolation of the two different directions yield another direction vector for which there is a canonical LSP from $p$ to $e_{\ell}$. The same holds for two canonical LSPs with adjacent direction vectors $\mathbf{v}$ and $\mathbf{w}$ from $e_{\ell-2^{i}}$ to the same point in $e_{\ell}$. 
The construction of $\mathcal{L}_{\alpha}^{i}$ proceeds in increasing $i$. The base case is $\mathcal{L}_{\alpha}^{0}$. Let $H_{\ell}$ denote the convex polygon that induces the distance function for the face bounded by $e_{\ell-1}$ and $e_{\ell}$. $\mathcal{L}_{\alpha}^{0}$ consists of the direction vector $\left(\hat{e}_{\ell-1}, \hat{e}_{\ell}\right)$ or $\left(\hat{e}_{\ell-1}, \hat{e}_{\ell}\right)$ depending on whether $e_{\ell-1}$ and $e_{\ell}$ share a negative or positive endpoint, respectively, and every vector consisting of a single direction that points to a vertex of $H_{\ell}$ and can be used to go from $e_{\ell-1}$ to $e_{\ell}$. For $i>0$, let $\beta$ be the ancestor edge-node of $\alpha$ at level $\ell-2^{i-1}$, and let $\left(\mathbf{u}_{1}, \ldots, \mathbf{u}_{r}\right)$ and $\left(\mathbf{v}_{1}, \ldots, \mathbf{v}_{r^{\prime}}\right)$ be the sequences of direction vectors in $\mathcal{L}_{\beta}^{i-1}$ and $\mathcal{L}_{\alpha}^{i-1}$, respectively. $\mathcal{L}_{\beta}^{i-1}$ has been computed as $\beta$ is at tree level $\ell-2^{i-1}$ and $2^{i}$ divides $\ell$. Choose an arbitrary point $p \in I_{\beta, \mathbf{u}_{k}} \cap I_{\beta, \mathbf{u}_{k+1}}$.

For all $k \in[1, r-1]$, take an arbitrary $p \in I_{\beta, \mathbf{u}_{k}} \cap I_{\beta, \mathbf{u}_{k+1}}$ and compute $\lambda_{\beta, k}=\frac{A_{\beta, \mathbf{u}_{k+1}}(p)-A_{\beta, \mathbf{u}_{k}}(p)}{\left\|a_{\beta, \mathbf{u}_{k}}(p) a_{\beta, \mathbf{u}_{k+1}}(p)\right\|}$. By P3, $\lambda_{\beta, k}$ equals $\partial C_{p, \sigma}^{+}$at $a_{\beta, \mathbf{u}_{k}}(p)$, where $\sigma=\left(e_{\ell-2^{i}+1}, \ldots, e_{\ell-2^{i-1}}\right)$, which is consistent with Lemma 3.6: $\partial C_{p, \sigma}^{+}$is independent of the source $p$ and the destination. Similarly, for $k \in\left[1, r^{\prime}-1\right]$, take an arbitrary $q \in a_{\alpha, \mathbf{v}_{k}}\left[I_{\alpha, \mathbf{v}_{k}}\right] \cap a_{\alpha, \mathbf{v}_{k+1}}\left[I_{\alpha, \mathbf{v}_{k+1}}\right]$ and compute $\pi_{\alpha, k}=\frac{B_{\alpha, \mathbf{v}_{k+1}}(q)-B_{\alpha, \mathbf{v}_{k}}(q)}{\left\|b_{\alpha, \mathbf{v}_{k}}(q) b_{\alpha, \mathbf{v}_{k+1}}(q)\right\|}$, which equals $\partial D_{q, \sigma}^{+}$at $b_{\alpha, \mathbf{v}_{k}}(q)$. By Lemma 3.3, $\mathcal{L}_{\alpha}^{i}$ consists of every concatenation $\mathbf{u}_{j} \mathbf{v}_{k}$ such that $\lambda_{\beta, j}+\pi_{\alpha, k} \geq 0$ and $\lambda_{\beta, j-1}+\pi_{\alpha, k-1}<0$. By Lemma 3.2, $\lambda_{\beta, j} \leq \lambda_{\beta, j+1}$ and $\pi_{\alpha, k} \leq \pi_{\alpha, k+1}$, so we can scan $\lambda_{\beta, j}$ in increasing $j$ and $\pi_{\alpha, k}$ in decreasing $k$ to identify the good concatenations. We first find $k_{0} \in\left[1, r^{\prime}\right]$ such that $\lambda_{\beta, 1}+\pi_{\alpha, k_{0}} \geq 0$ and $\lambda_{\beta, 1}+\pi_{\alpha, k_{0}-1}<0$, and so $\mathbf{u}_{1} \mathbf{v}_{k_{0}}$ is a good concatenation. Note that $\lambda_{\beta, 1}+\pi_{\alpha, k}<0$ for all $k<k_{0}$. Next, we find $k_{1} \leq k_{0}$ such that $\lambda_{\beta, 2}+\pi_{\alpha, k_{1}} \geq 0$ and $\lambda_{\beta, 2}+\pi_{\alpha, k_{1}-1}<0$. Thus, $\lambda_{\beta, 2}+\pi_{\alpha, k} \geq 0$ and $\lambda_{\beta, 1}+\pi_{\alpha, k-1}<$ 0 for all $k \in\left[k_{1}, k_{0}\right]$, which makes $\mathbf{u}_{2} \mathbf{v}_{k}$ a good concatenation for all $k \in\left[k_{1}, k_{0}\right]$. Repeating the above gives $\mathcal{L}_{\alpha}^{i}$. When adding a concatenation uv, we compute in $O(1)$ time $I_{\alpha, \mathbf{u v}}=b_{\beta, \mathbf{u}}\left[a_{\beta, \mathbf{u}}\left[I_{\beta, \mathbf{u}}\right] \cap I_{\alpha, \mathbf{v}}\right], A_{\alpha, \mathbf{u v}}=A_{\beta, \mathbf{u}}+A_{\alpha, \mathbf{v}} \circ a_{\beta, \mathbf{u}}$, $a_{\alpha, \mathbf{u v}}=a_{\alpha, \mathbf{v}} \circ a_{\beta, \mathbf{u}}, B_{\alpha, \mathbf{u v}}=B_{\alpha, \mathbf{v}}+B_{\beta, \mathbf{u}} \circ b_{\alpha, \mathbf{v}}$, and $b_{\alpha, \mathbf{u v}}=$ $b_{\beta, \mathbf{u}} \circ b_{\alpha, \mathbf{v}}$, where the operator $\circ$ composes two functions.

Lemma 3.9. For every $i \geq 0, \mathcal{L}_{\alpha}^{i}$ satisfies $\mathrm{P} 1-\mathrm{P} 4$.

Proof. Consider the base case of $i=0$. P1 holds because any direction vector added has only one link. P2 holds by the choices of directions picked by the algorithm. Suppose that $\mathbf{v}_{j}=\left(\hat{v}_{j}\right)$ and $\mathbf{v}_{j+1}=\left(\hat{v}_{j+1}\right)$ are two adjacent direction vectors. The direction of the oriented segment from $p$ to any point between $a_{\alpha, \mathbf{v}_{j}}(p)$ and $a_{\alpha, \mathbf{v}_{j+1}}(p)$ lies between $\hat{v}_{j}$ and $\hat{v}_{j+1}$. Since $\hat{v}_{j}$ and $\hat{v}_{j+1}$ point to the same edge of the convex polygon that defines the distance function, the cost of the segment from $p$ to a point between $a_{\alpha, \mathbf{v}_{j}}(p)$ and $a_{\alpha, \mathbf{v}_{j+1}}(p)$ is a linear interpolation of $A_{\alpha, \mathbf{v}_{j}}(p)$ and $A_{\alpha, \mathbf{v}_{j+1}}(p)$. Thus, P3 holds. P4 can be proved similarly.

Consider the case of $i>0$. Let $\ell$ be the level of $\alpha$. Let $\beta$ be the ancestor edge-node of $\alpha$ at level $\ell-2^{i-1}$. Assume that P1-P4 hold for both $\mathcal{L}_{\alpha}^{i-1}$ and $\mathcal{L}_{\beta}^{i-1}$. P1 holds for $\mathcal{L}_{\alpha}^{i}$ by our method to identify good concatenations.

Consider P2. Any two successive concatenations added to $\mathcal{L}_{\alpha}^{i}$ share either a prefix, i.e. $\mathbf{u v}$ and $\mathbf{u v}^{\prime}$, or a suffix, i.e. $\mathbf{u v}$ and $\mathbf{u}^{\prime} \mathbf{v}$. By P2, $\mathbf{u}$ and $\mathbf{u}^{\prime}$ differ in exactly one entry, and so do $\mathbf{v}$ and $\mathbf{v}^{\prime}$. It follows that $\mathcal{L}_{\alpha}^{i}$ satisfies P2.

Consider P3. Take any two adjacent direction vectors in $\mathcal{L}_{\alpha}^{i-1}$ and $\mathcal{L}_{\beta}^{i-1}$. They differ in one entry by P2 and we can write them as $\mathbf{u}=\mathbf{w}\left(\hat{w}_{0}\right) \mathbf{w}^{\prime}$ and $\mathbf{v}=\mathbf{w}\left(\hat{w}_{1}\right) \mathbf{w}^{\prime}$. Let $P$ and $Q$ be the canonical LSPs from $p$ to $a_{\alpha, \mathbf{u}}(p)$ and $a_{\alpha, \mathbf{v}}(p)$ respectively. Consider the canonical LSP $R$ from $p$ to a point $q=(1-t) a_{\alpha, \mathbf{u}}(p)+t a_{\alpha, \mathbf{v}}(p)$ for some $t \in[0,1]$. By Lemma 3.3, the direction vector of $R$ is $\mathbf{w}(\hat{w}) \mathbf{w}^{\prime}$, where $\hat{w}$ lies between $\hat{w}_{0}$ and $\hat{w}_{1}$. Let $r x, r y$ and $r z$ be the segments of $P, R$ and $Q$, respectively, that have directions $\hat{w}_{0}$, $\hat{w}$ and $\hat{w}_{1}$, respectively. Because $R[y, q], P\left[x, a_{\alpha, \mathbf{u}}(p)\right]$ and $Q\left[z, a_{\alpha, \mathbf{v}}(p)\right]$ have the same direction vector, we get $y=$ $(1-t) x+t z$, and $\operatorname{cost}(R[y, q])=(1-t) \operatorname{cost}\left(P\left[x, a_{\alpha, \mathbf{u}}(p)\right]\right)+$ $t \operatorname{cost}\left(Q\left[z, a_{\alpha, \mathbf{v}}(p)\right]\right)$. By P2, $\operatorname{cost}(r y)=(1-t) \operatorname{cost}(r x)+$ $t \operatorname{cost}(r z)$. Therefore, $\operatorname{cost}(R)=(1-t) \operatorname{cost}(P)+t \operatorname{cost}(Q)$.

$\mathrm{P} 4$ can be proved similarly.

Lemma 3.10. An edge-node at level $\ell$ takes $O\left(2^{i} h\right)$ time to create, where $2^{i}$ is the largest power of 2 that divides $\ell$, and $h$ is the maximum size of the convex polygons associated with the faces.

Proof. Let $\alpha$ be a new edge-node at level $\ell . \mathcal{L}_{\alpha}^{0}$ stores $O(h)$ direction vectors. For $i>0$, let $\beta$ be the edge-node at level $\ell-2^{i-1}$, the size of $\mathcal{L}_{\alpha}^{i}$ is at most the total size of $\mathcal{L}_{\alpha}^{i-1}$ and $\mathcal{L}_{\beta}^{i-1}$. Inductively, we obtain a time bound of $\sum_{j=0}^{i-1} O\left(2^{j} h\right)=O\left(2^{i} h\right)$.

\subsubsection{Compute an LSP to a vertex}

Suppose that we expand an edge-node $\alpha$ at tree level $\ell$ that annexes a face corner $(f, \nu)$. Let $\alpha_{0}$ be the nearest ancestor vertex-node of $\alpha$ at tree level $\ell_{0}<\ell$. Let $\left(e_{\ell_{0}+1}, \ldots, e_{\ell}\right)$ be the edge sequence corresponding to the edge-nodes on the tree path from $\alpha_{0}$ to $\alpha$. We are to create a vertex-node $\beta$ for $\nu$ and compute the cost of the canonical LSP $P_{\beta}$ from $s$ to $\nu$ through the edges $\left(e_{\ell_{0}+1}, \ldots, e_{\ell}\right)$.

For $i=1,2, \ldots$, find the largest $\ell_{i}$ such that $\ell_{i} \leq \ell$ and $\ell_{i}-\ell_{i-1}$ is a power of 2 that divides both $\ell_{i}$ and $\ell_{i-1}$. This gives a sequence $\ell_{0}<\ell_{1}<\ldots<\ell_{r}=\ell$, where $r=O(\log \ell)$. For $i \geq 1$, let $k_{i}=\ell_{i}-\ell_{i-1}$ and let $\alpha_{i}$ be the ancestor edgenode of $\alpha$ at level $\ell_{i}$. We also use $\alpha_{r}$ to denote $\alpha$. Let $\sigma_{i}$ denote the edge sequence $\left(e_{\ell_{0}+1}, \ldots, e_{\ell_{i}}\right)$ for $i \in[1, r]$. $P_{\beta}$ is the concatenation of $P_{\alpha_{0}}$ and the canonical LSP $P$ from $\nu_{\alpha_{0}}$ to $\nu$ through the edges $\left(e_{\ell_{0}+1}, \ldots, e_{\ell}\right)$. We already know $\operatorname{cost}\left(P_{\alpha_{0}}\right)$. We compute $\operatorname{cost}(P)$ by combining the $\mathcal{L}_{\alpha_{i}}^{k_{i}}$ 's in at most $r+1$ stages. At the end of the $i$-th stage, $i \in[1, r]$, we fix the prefix $Q_{i}$ of $P$ up to $e_{\ell_{i}}{ }^{5}$

Assume that $Q_{i-1}$ is fixed. Let $x_{i-1}$ denote its destination. Assume that we have computed $\partial C_{\nu_{\alpha_{0}}, \sigma_{i-1}}^{+}\left(x_{i-1}\right)$ and $\partial C_{\nu_{\alpha_{0}}, \sigma_{i-1}}^{-}\left(x_{i-1}\right)$. Consider the canonical LSPs from $\nu_{\alpha_{0}}$ through $\left(e_{\ell_{i-1}+1}, \ldots, e_{\ell}\right)$ with $Q_{i-1}$ as a common prefix. By Lemmas 3.2 and 3.7, these LSPs spread out from $x_{i-1}$ to $e_{\ell}$ and form a fan that contains $\nu_{\alpha}$. We want to construct a path $R$ from $x_{i-1}$ to a point $y \in e_{\ell_{i}}$ such that $Q_{i}=Q_{i-1} R$ and the canonical LSPs that spread out from $y$ to $e_{\ell}$ form a fan that contains $\nu_{\alpha}$. Let $\mathbf{u}_{i-1}$ be the direction vector of $Q_{i-1}$. The idea is to find the direction vector $\mathbf{v}$ in $\mathcal{L}_{\alpha_{i}}^{k_{i}}$ by binary search such that $\mathbf{u v}$ is the direction vector of $Q_{i}$.

The binary search works as follows. Let $\mathbf{v}$ be the "median" direction vector in the sublist of $\mathcal{L}_{\alpha_{i}}^{k_{i}}$ that we are working on. If $x_{i-1} \notin I_{\alpha_{i}, \mathbf{v}}$, we remove half of the sublist of $\mathcal{L}_{\alpha_{i}}^{k_{i}}$ and recurse. Suppose that $x_{i-1} \in I_{\alpha_{i}, \mathbf{v}}$. We find the smallest direction vector $\mathbf{w}$ and the largest direction vector $\mathbf{w}^{\prime}$ such that $\mathbf{u}_{i-1} \mathbf{v w}$ and $\mathbf{u}_{i-1} \mathbf{v} \mathbf{w}^{\prime}$ extend $Q_{i-1}$ to two canonical LSPs through $\left(e_{\ell_{i-1}+1}, \ldots, e_{\ell}\right)$ and the face $f$. (We will

${ }^{5}$ We define the prefix $Q_{r}$ of $P$ only up to $e_{\ell_{r}}=e_{\ell}$ instead of an edge of $f$ incident to $\nu$. It is because we will apply Lemmas 3.4-3.6, which require the nodes the path other than its source to be in the interior of edges. 
describe how to find $\mathbf{w}$ and $\mathbf{w}^{\prime}$ shortly.) If these two LSPs lie on the same side of $\nu_{\alpha}$, by Lemma 3.7, we can remove half of the sublist of $\mathcal{L}_{\alpha_{i}}^{k_{i}}$ and recurse. If these two LSPs sandwich $\nu_{\alpha}$, then $\mathbf{u}_{i-1} \mathbf{v}$ extends $Q_{i-1}$ to $Q_{i}$. The destination of $Q_{i}$ is $y=a_{\alpha_{i}, \mathbf{v}}\left(x_{i-1}\right)$ and $\operatorname{cost}\left(Q_{i}\right)=\operatorname{cost}\left(Q_{i-1}\right)+A_{\alpha_{i}, \mathbf{v}}\left(x_{i-1}\right)$. By Lemmas 3.4-3.6, $\partial C_{\nu_{\alpha_{0}}, \sigma_{i}}^{+}(y)$ and $\partial C_{\nu_{\alpha_{0}}, \sigma_{i}}^{-}(y)$ can be computed in $O(1)$ time. Then we fix the next prefix $Q_{i+1}$. If we proceed all the way to stage $r$ and fix $Q_{r}$, then $\operatorname{cost}(P)=$ $\operatorname{cost}\left(Q_{r}\right)+\operatorname{cost}\left(x_{r} \nu\right)$, where $x_{r}$ is the destination of $Q_{r}$. The binary search may also finish with two adjacent direction vectors in $\mathcal{L}_{\alpha_{i}}^{k_{i}}$ without fixing $Q_{i}$, a terminating case that we discuss after the next paragraph.

How do we find the smallest and largest direction vectors $\mathbf{w}$ and $\mathbf{w}^{\prime}$ ? By Lemma 3.3, we find the smallest direction vector $\mathbf{w}_{i+1}$ in $\mathcal{L}_{\alpha_{i+1}}^{k_{i+1}}$ by binary search such that $\mathbf{v w}_{i+1}$ is the direction vector of some canonical LSP from $x_{i-1}$ through $\left(e_{\ell_{i-1}+1}, \ldots, e_{\ell_{i+1}}\right)$. Let $y=a_{\alpha_{i}, \mathbf{v}}\left(x_{i-1}\right)$ and let $z=a_{\alpha_{i+1}, \mathbf{w}_{i+1}}(y)$. We apply Lemmas 3.4-3.6 to compute $\partial C_{\nu_{\alpha_{0}}, \sigma_{i+1}}^{+}(z)$ and $\partial C_{\nu_{\alpha_{0}}, \sigma_{i+1}}^{-}(z)$ in $O(1)$ time. Then, we find the smallest direction vector $\mathbf{w}_{i+2}$ in $\mathcal{L}_{\alpha_{i+2}}^{k_{i+2}}$ by binary search and extend to $\mathbf{v} \mathbf{w}_{i+1} \mathbf{w}_{i+2}$. Repeating the above gives $\mathbf{w}_{i+1} \mathbf{w}_{i+2} \ldots \mathbf{w}_{r}$. Finally, we pick the smallest direction $\hat{w}_{r+1}$ according to Lemma 3.3 that extends $\mathbf{w}_{i+1} \mathbf{w}_{i+2} \ldots \mathbf{w}_{r}$ through $f$, and $\mathbf{w}_{i+1} \mathbf{w}_{i+2} \ldots \mathbf{w}_{r}\left(\hat{w}_{r+1}\right)$ is the desired $\mathbf{w}$. The largest direction vector $\mathbf{w}^{\prime}$ is obtained symmetrically.

Recall the terminating case that $Q_{i}$ cannot be fixed and the binary search finishes with two adjacent direction vectors $\mathbf{v}$ and $\mathbf{v}^{\prime}$ in $\mathcal{L}_{\alpha_{i}}^{k_{i}}$. We find the largest direction vector $\mathbf{w}$ as before to extend $\mathbf{v}$ through $\left(e_{\ell_{i}+1}, \ldots, e_{\ell}\right)$ and $f$. Note that $\mathbf{v w}$ and $\mathbf{v}^{\prime} \mathbf{w}$ extend $Q_{i-1}$ to two canonical LSPs that sandwich $\nu$, so $\mathbf{w}$ is the direction vector of the subpath of $P$ from $e_{\ell_{i}}$ to $\nu$. The last direction $\hat{w}_{r+1}$ in $\mathbf{w}$ brings us from $\nu$ to a point $z \in e_{\ell}$, and the cost is $\operatorname{cost}(z \nu)$. We continue to $b_{\alpha_{r}, \mathbf{w}_{r}}(z) \in e_{\ell_{r-1}}$ and the cost accumulates to $\operatorname{cost}(z \nu)+$ $B_{\alpha_{i}, \mathbf{w}_{r}}(z)$, and so on to a point $y \in e_{\ell_{i}}$ between $a_{\alpha_{i}, \mathbf{v}}\left(x_{i-1}\right)$ and $a_{\alpha_{i}, \mathbf{v}^{\prime}}\left(x_{i-1}\right)$, where $x_{i-1}$ is the destination of $Q_{i-1}$. Let $C$ be the cost of the path that we have retraced from $\nu$ to $e_{\ell_{i}}$. Suppose that $y=(1-t) a_{\alpha_{i}, \mathbf{v}}\left(x_{i-1}\right)+t a_{\alpha_{i}, \mathbf{v}^{\prime}}\left(x_{i-1}\right)$. By P3 and Lemma $3.3, \operatorname{cost}(P)=\operatorname{cost}\left(Q_{i-1}\right)+(1-t) A_{\alpha_{i}, \mathbf{v}}\left(x_{i-1}\right)+$ $t A_{\alpha_{i}, \mathbf{v}^{\prime}}\left(x_{i-1}\right)+C$.

TheOREM 3.1. Let $\mathcal{T}$ be a polyhedral surface with $n$ vertices in an instance of POLYPATH. Given a source $s$, a destination $t$ and an integer $m$, the shortest path from $s$ to $t$ on $\mathcal{T}$ with no more than $m$ links can be found in $O(h m n \log m n+$ $\left.m n \log ^{2} m \log ^{2} h m\right)$ time, where $h$ is the maximize size of the convex polygons that define the distance functions in the faces of $\mathcal{T}$.

Proof. Consider the correctness of the algorithm. Let $P_{0}$ be the shortest path from $s$ to $t$ with no more than $m$ links. By the requirement of the PoLYPATH problem, we can assume that every node of $P_{0}$ is either a transversal node or a vertex of $\mathcal{T}$. If there are multiple choices for $P_{0}$, we pick $P_{0}$ to be one that has the fewest nodes.

The sequence tree is grown to contain the prefix of $P_{0}$ until the vertex-node corresponding to $t$ is reached or an edgenode $\alpha_{0}$ is dominated by some other edge-node $\beta$ such that the child node of $\alpha_{0}$ that would contain a longer prefix of $P_{0}$ is pruned. In the former case, the sequence tree captures the edge sequence of $P_{0}$, and the algorithm computes the cost of the LSP with respect to that edge sequence, so we are done. Consider the latter case. Let $x$ be the intersection point between $P_{0}$ and the edge corresponding to $\alpha_{0}$ and $\beta$. By Lemma 3.8, there exists a path $Q$ from $s$ to $x$ with edge sequence $\sigma_{\beta}$ that is at least as good as $P[s, x]$. Let $P_{1}=Q \cdot P[x, t]$. By our choice of $P_{0}, \operatorname{cost}\left(P_{1}\right)=\operatorname{cost}\left(P_{0}\right)$, $P_{1}$ has the same number of nodes as $P_{0}$, and $\beta$ is at the same depth as $\alpha_{0}$ but expanded earlier. Note that $\beta$ cannot be dominated by any other edge-node. The subtree of $\beta$ grows to contain $P_{1}$, or a descendant $\alpha_{1}$ of $\beta$ is dominated by some other edge-node and the child of $\alpha_{1}$ that would contain a longer prefix of $P_{1}$ is pruned. We can then repeat the analysis above, which can happen at most $m$ times. The correctness thus follows.

Consider the running time. We spend $O(\log m n)$ amortized time in each invocation of dominance testing and pruning. So we create $O(m n)$ tree nodes in $O(m n \log m n)$ time.

Divide the edge-nodes into $O(\log m)$ groups such that an edge-node is in group $i$ if its level is a multiple of $2^{i}$ but not $2^{i+1}$. Group $i$ contains $O\left(m n / 2^{i}\right)$ edge-nodes. By Lemma 3.10, creating a node in group $i$ takes $O\left(2^{i} h\right)$ time. So it takes $O(h n m \log m)$ time to create all the edge-nodes.

To compute the cost of an LSP for a vertex-node, we fix $O(\log m)$ prefixes $Q_{i}$ 's. To extend $Q_{i-1}$ to $Q_{i}$, we binary search in $\mathcal{L}_{\alpha_{i}}^{k_{i}}$ in $O\left(\log 2^{i} h\right)=O(\log \ell h)=O(\log h m)$ probes by Lemma 3.10. Each probe requires $O(\log \ell)=$ $O(\log m)$ binary searches among the lists $\mathcal{L}_{\alpha_{i+1}}^{k_{i+1}}, \mathcal{L}_{\alpha_{i+2}}^{k_{i+2}}, \ldots$ So it takes $O\left(\log ^{2} m \log ^{2} h m\right)$ time to compute the cost of an LSP. The total time spent on all vertex-nodes is thus $O\left(m n \log ^{2} m \log ^{2} h m\right)$. We can reconstruct the direction vector of the shortest path in a similar way as in dealing with the terminating case of not fixing some $Q_{i}$. So constructing the path takes only $O(m)$ time.

\section{APPLICATIONS}

Under the $L_{1}$ and $L_{\infty}$ metrics, $h=O(1)$. Under the $L_{p}$ metric for some $p \geq 2$, Dudley's result [11] allows us to approximate the "unit disk" by a polygon of $O(1 / \sqrt{\varepsilon})$ vertices such that the polygon diameter is approximated with an $\varepsilon$ relative error, i.e., $h=O(1 / \sqrt{\varepsilon})$. In the above cases, $m=O(n)$ because there exists a shortest path that visits a face no more than once.

THEOREM 4.1. Given a polyhedral surface of size $n$, the $L_{1}$ and $L_{\infty}$ shortest paths between two vertices can be computed in $O\left(n^{2} \log ^{4} n\right)$ time, and for every constant $p \geq 2$ and every $\varepsilon \in(0,1)$, a $(1+\varepsilon)$-approximate $L_{p}$ shortest path can be computed in $O\left(\frac{1}{\sqrt{\varepsilon}} n^{2} \log n+n^{2} \log ^{4} n\right)$ time.
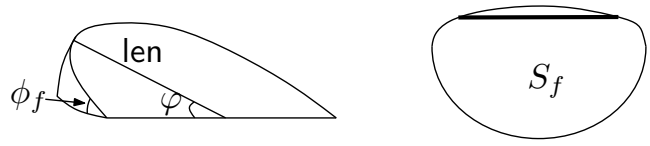

Figure 5: Left: The face $f$ makes an angle $\phi_{f}$ with the horizontal, and the ascent is len $\cdot \sin \varphi \sin \phi_{f}$. Right: The bold segment represents the clipping.

Consider path planning on a terrain with the cost function $c_{1}$. Euclidean length $+c_{2}$. total ascent for some constants $c_{1}>0$ and $c_{2} \geq 0$. Refer to the left image in Figure 5 . The ascent within a face $f$ is len $\cdot \sin \varphi \sin \phi_{f}$, where len is the distance travelled in $f, \phi_{f}$ is the gradient of a face 
$f$, and $\varphi$ is the angle between the travel direction and the horizontal. Let $S_{f}$ denote the "unit disk" induced. On the uphill side, the boundary of $S_{f}$ satisfies the equation $1=$ $\left(c_{1}+c_{2} \sin \varphi \sin \phi_{f}\right)$ len; on the downhill side, the boundary of $S_{f}$ is the half-circle with radius $1 / c_{1} . S_{f}$ is convex with bounded aspect ratio, so we can approximate it by Dudley's result [11] to obtain a POLYPATH problem instance with $h=$ $O(1 / \sqrt{\varepsilon})$ and $m=O(n)$.

We can incorporate uphill gradient constraints. Let $\psi$ be the input limit on the uphill path gradient. Let $p q$ be an oriented segment in the interior of $f$ that makes an angle larger than $\psi$ with the horizontal. We can traverse a zigzag path within $f$ from $p$ to $q$ in which each segment makes an angle $\psi$ with the horizontal. The path length is equal to the height difference between $p$ and $q$ divided by $\sin \psi$, irrespective of the exact zigzag pattern. Under this constraint, the top part of $S_{f}$ that makes an angle at least $\psi$ with the horizontal should be clipped. Refer to the right image in Figure 5. We can similarly handle downhill gradient constraints. Note that such a zigzag is treated as a "single" link.

There are some technical issues. Let $P$ be a shortest path from $s$ to $t$ that satisfies the gradient constraints. Consider the intersections between $P$ and a face $f$. Let $p$ be the first point of entry. Let $q$ be the last point of exit. Suppose that neither $p$ nor $q$ is a vertex of $f$. Without loss of generality, assume that $q$ is higher than $p$. Let $\psi$ be the ascent gradient bound. We assume that the gradient of $p q$ exceeds $\psi$; otherwise, we can connect $p$ and $q$ directly. Let $p_{0}$ and $q_{0}$ be in the interior of $f$ arbitrarily close to $p$ and $q$, respectively, such that $p p_{0}$ and $q_{0} q$ satisfy the gradient constraints. We can follow $p p_{0}$, then a zigzag path from $p_{0}$ to $q_{0}$ and then $q_{0} q$ to reach $q$ from $p$ with constant ascent gradient $\psi$. The lengths of segments $p p_{0}$ and $q_{0} q$ are negligible, so the length of this path is $H /(\sin \psi)$, where $H$ is the height difference between $p$ and $q$. The subpath of $P$ from $p$ to $q$ cannot be shorter because the same height difference $H$ is covered with a ascent gradient no greater than $\psi$. At the same time, the ascent of the zigzag path is the smallest possible because it goes monotonically upward. If $q$ is a vertex of $f$, it may be impossible to move from any point in $f$ straight to $q$. After $O(n)$-time preprocessing, for every vertex, we can determine if it can be reached from some point in its close neighborhood under the gradient constraints. If there is a point $q^{\prime \prime}$ in a face $f^{\prime \prime}$ incident to $q$ such that $q^{\prime \prime} q$ satisfies the gradient constraints, then we can first move from $p$ to a point $q^{\prime} \in f$ near $q$ using a zigzag path such that every segment of the zigzag path has uphill gradient $\psi$, then to $q^{\prime \prime}$ and then to $q$. We can make $q^{\prime}$ and $q^{\prime \prime}$ arbitrarily close to $q$, so the detour cost can be made negligible. A similar detour may be needed for $p$ if it is also a vertex.

This gives an instance of POLYPATH with $h=O(1 / \sqrt{\varepsilon})$ and $m=O(n)$. The algorithm need to be slightly modified due to the technical issues we mentioned earlier. If a vertex cannot be reached locally, then we never create the corresponding vertex-node in the sequence tree. If no path can be extended from from a vertex, then we do not expand the corresponding vertex-node.

THEOREM 4.2. Given a source $s$ and a destination $t$ on a polyhedral terrain of size $n$, we can find $a(1+\varepsilon)$-approximate shortest path under the cost function of $c_{1}$ length $+c_{2}$ ascent for some constants $c_{1}>0$ and $c_{2} \geq 0$, where length is the Euclidean path length and ascent is the total ascent.
Gradient constraints can be imposed. The running time is $O\left(\frac{1}{\sqrt{\varepsilon}} n^{2} \log n+n^{2} \log ^{4} n\right)$.

\section{REFERENCES}

[1] M. Ahmed, S. Das, S. Lodha, A. Lubiw, A. Maheshwari and S. Roy. Approximation algorithms for shortest descending paths in terrains. J. Discrete Alg., 8 (2010), 214-230.

[2] M. Ahmed and A. Lubiw. Shortest descending paths: towards an exact algorithm. Internat. J. Comput. Geom. Appl., 21 (2011), 431-466.

[3] M. Ahmed, A. Lubiw, and A. Maheshwari. Shortest gently descending paths. WALCOM, 2009, 59-70.

[4] L. Aleksandrov, A. Maheshwari, and J.-R. Sack. Determining approximate shortest paths on weighted polyhedral surfaces. J. ACM, 52 (2005), 25-53.

[5] M. de Berg and M. van Kreveld. Trekking in the Alps without freezing or getting tired. Algorithmica, 18 (1997), 306-323.

[6] J. Chen and Y. Han. Shortest paths on a polyhedron, part I: computing shortest paths. Internat. J. Comput. Geom. Appl., 6 (1996), 127-144.

[7] S.-W. Cheng and J. Jin. Approximate shortest descending paths. Proc. 24th Annu. ACM-SIAM Sympos. Discrete Alg., 2013, 144-155.

[8] S.-W. Cheng, H.-S. Na, A. Vigneron, and Y. Wang. Approximate shortest paths in anisotropic regions. SIAM J. Comput., 38 (2008), 802-824.

[9] S.-W. Cheng, H.-S. Na, A. Vigneron, and Y. Wang. Querying approximate shortest paths in anisotropic regions. SIAM J. Comput., 39 (2010), 1888-1918.

[10] W. Collischonn and J.V. Pilar. A direction dependent least-costs path algorithm for roads and canals. Internat. J. Geo. Info. Sci., 14 (2000), 391-406.

[11] R. M. Dudley. Metric entropy of some classes of sets with differentiable boundaries. Approx. Theory, 10 (1974), 227-236.

[12] L. Liu and R. C.-W. Wong. Finding shortest path on land surface. SIGMOD'11, 433-444.

[13] J.S.B. Mitchell, D.M. Mount and C.H. Papadimitriou. The discrete geodesic problem. SIAM J. Comput., 16 (1987), 647-668.

[14] J.S.B. Mitchell and C.H. Papadimitriou. The weighted region problem: finding shortest paths through a weighted planar subdivision. J. ACM, 38 (1991), $18-73$.

[15] J.A. Roles and H. ElAarag. A smoothest path algorithm and its visualization tool. Proc. IEEE, 2013, $1-6$

[16] K.R. Varadarajan and P.K. Agarwal. Approximating shortest paths on a non-convex polyhedron. SIAM J. Comput., 30 (2001), 1321-1340.

[17] C. Yu, J. Lee, and M.J. Munro-Stasiuk. Extensions to least-cost path algorithms for roadway planning. Internat. J. Geo. Info. Sci., 17 (2003), 361-376. 